\title{
Jahn-Teller effects in molecular cations studied by photoelectron spectroscopy and group theory
}

\section{Journal Article}

Author(s):

Wörner, Hans Jakob; Merkt, Frédéric

Publication date:

2009-08-17

Permanent link:

https://doi.org/10.3929/ethz-a-010782632

Rights / license:

In Copyright - Non-Commercial Use Permitted

Originally published in:

Angewandte Chemie. International Edition 48(35), https://doi.org/10.1002/anie.200900526 
This article may be downloaded for personal use only. Any other use requires prior permission of the author and John Wiley \& Sons, Inc..

The following article appeared in Angew. Chemie Int. Ed. 48, 6404-6424 (2009) and may be found at http://dx.doi.org/10.1002/anie.200900526. 
Jahn-Teller effects in molecular cations studied by photoelectron spectroscopy and group theory *

Hans Jakob Wörner and Frédéric Merkt

Laboratorium für Physikalische Chemie, ETH-Zürich,

8093 Zürich, Switzerland

28th March 2009

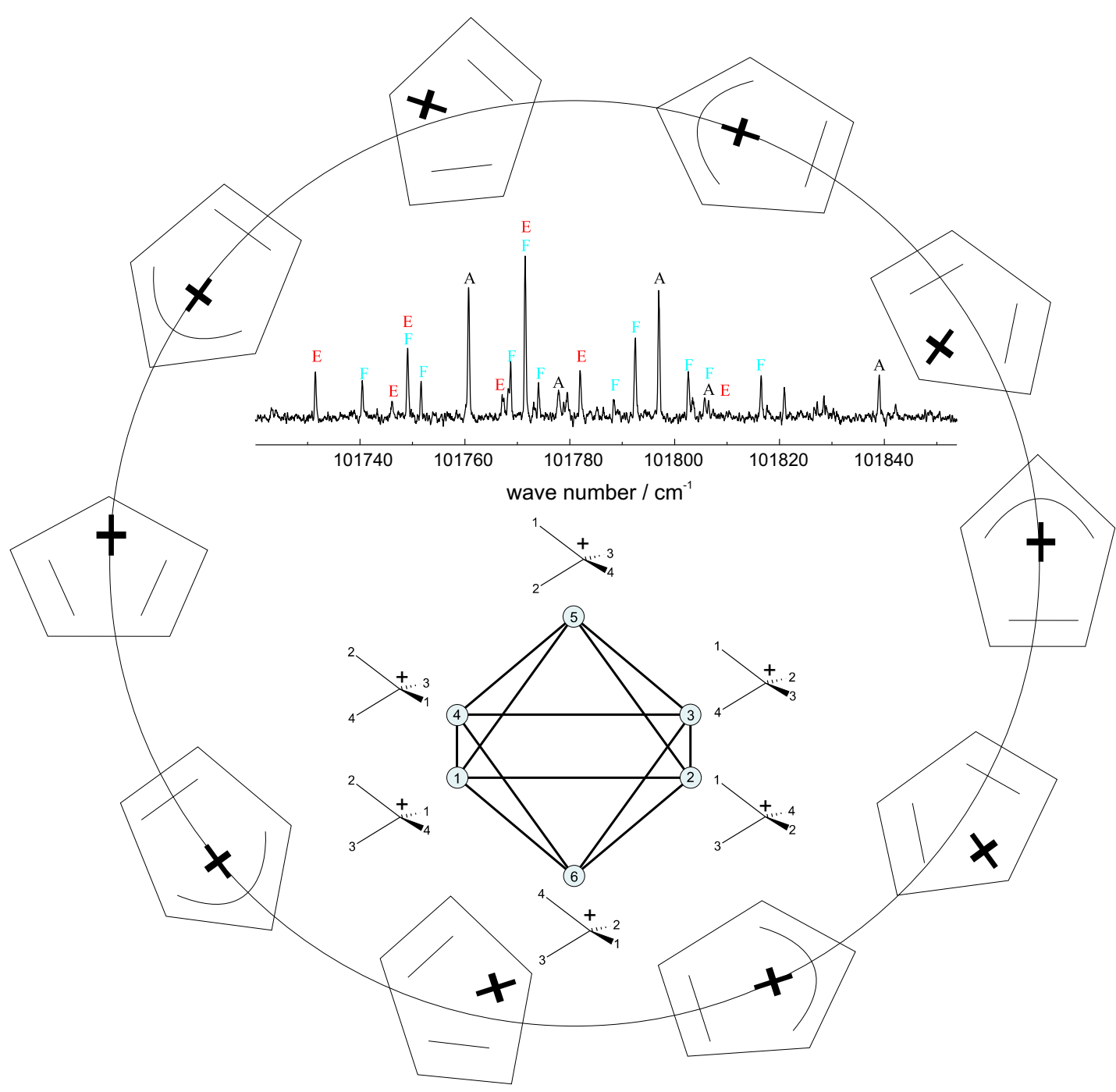

${ }^{*}$ dedicated to Prof. Martin Quack on the occasion of his $60^{\text {th }}$ birthday 
Understanding the structure and dynamics of molecules is the most fundamental goal of chemistry. The traditional "balls-and-sticks" concept of molecular structure fails when the motion of the electrons is coupled to that of the nuclei. Such a situation arises in the Jahn-Teller (JT) effect which is very common in open-shell molecular systems, such as radicals or ions. The JT effect is well known to chemists as a mechanism that causes the distortion of an otherwise symmetric system. Its implications on the dynamics still represent unsolved problems in many molecules. This article reviews recent progress in understanding the dynamic structure of molecular cations having a high permutational symmetry using rotationally resolved photoelectron spectroscopy and group theory. The JT effect profoundly modifies their electronic structure and renders them fluxional. Specifically, we show how the Pseudo-JT effect in the cyclopentadienyl cation causes electronic localization and nuclear delocalization. The fundamental physical mechanisms underlying the vaguely defined concept of "antiaromaticity" are thereby elucidated. Our investigation of the methane cation represents the first experimental characterization of the JT effect in a threefold degenerate electronic state. A special kind of isomerism resulting from the JT effect has been discovered and is predicted to exist in all JT systems in which the minima on the potential energy surface are separated by substantial barriers. The JT effect can also induce chirality which results in an interesting case of stereomutation dynamics.

\section{Introduction}

The Jahn-Teller (JT) and Pseudo-Jahn-Teller (PJT) effects arise from the coupling of electronic and nuclear degrees of freedom in manifolds of degenerate or near-degenerate states and affects the structural and dynamical properties of molecules, transition metal complexes and solids. The variety of its physical and chemical implications is extremely rich $[1,2]$. In electronically degenerate states of molecules and transition metal complexes, the JT effect lowers the potential energy for configurations of the nuclei having a reduced symmetry. If the corresponding JT stabilization energy is large compared to vibrational energy intervals, the JT effect results in a distortion of the molecule. In organic molecules, the JT effect is essential in the interpretation of photochemistry and in the definition of antiaromaticity. In solids, the JT effect has been invoked to explain phenomena like superconductivity and colossal magnetoresistance [1]. The JT effect occurs in species with unpaired electrons, i.e. radicals, biradicals, etc. Such molecules are usually highly reactive and play an important role as reaction intermediates, in combustion chemistry, atmospheric chemistry and the chemistry of the interstellar medium. 
The first advances in the investigation of the JT effect were of theoretical nature. Hermann Jahn and Edward Teller used group theory to prove that a nonlinear molecule in an orbitally degenerate state undergoes a geometric distortion [3,4]. Longuet-Higgins $[5,6]$ calculated the vibronic energy level structure resulting from the interaction of a doubly degenerate (E) electronic state with one doubly degenerate (e) vibrational mode, which is called the $\mathrm{E} \otimes \mathrm{e}$ JT effect. He predicted the band shapes of optical absorption spectra and pointed at the first observable manifestations of the JT effect in optical spectroscopy. A molecule subject to a JT effect can distort along several equivalent vibrational modes giving rise to equivalent minima on the potential energy surface. Bersuker recognized that this situation leads (in most cases) to a splitting of the lowest vibronic levels by tunneling [7]. Öpik and Pryce investigated the interaction of triply degenerate electronic states ( $\mathrm{T}$ ) and doubly (e) and triply (t) degenerate vibrational modes in molecules of the cubic point groups known as the $\mathrm{T} \otimes\left(\mathrm{e}+\mathrm{t}_{2}\right)$ effect and considered for the first time the vibronic coupling between nondegenerate electronic states, also called the Pseudo-Jahn-Teller (PJT) effect [8].

The most detailed experimental characterization of the JT effect has been achieved in highresolution optical and photoelectron spectroscopy. Optical spectroscopy has been applied to elucidate the JT effect in several open-shell species including $\mathrm{Na}_{3}$ and $\mathrm{Li}_{3}$. The $\tilde{\mathrm{A}}^{2} \mathrm{E}^{\prime \prime}$ state of these species represents the prototype of the $\mathrm{E} \otimes \mathrm{e}$ JT effect [9]. The next higher electronic state $\tilde{\mathrm{B}}^{2} \mathrm{~A}_{1}{ }^{\prime}$ is subject to a PJT effect $[10,11]$. Photoelectron spectroscopy has been used to characterize the JT effect in a wide range of molecular cations. For instance, the analysis of rotationally resolved PFI-ZEKE photoelectron spectra of $\mathrm{C}_{6} \mathrm{H}_{6}$ has proven that the minima of the ground state potential energy surface of $\mathrm{C}_{6} \mathrm{H}_{6}^{+}$, which is subject to the $\mathrm{E} \otimes \mathrm{e}$ JT effect, correspond to a $\mathrm{D}_{2 \mathrm{~h}}$ geometry with two acute $\mathrm{C}-\mathrm{C}-\mathrm{C}$ bond angles, although the rovibronic photoionization selection rules are adequately described in $\mathrm{D}_{6 \mathrm{~h}}(\mathrm{M})$ symmetry $[12,13]$.

Currently, the $\mathrm{E} \otimes \mathrm{e} \mathrm{JT}$ effect is the best understood case and its ramifications are well known [1,14-18]. Much less is known about other cases, especially about molecules of higher symmetry, where the electronic states can have threefold or higher degeneracies [19], primarily because of the scarcity of high-resolution spectroscopic data that would permit the determination of the structure and dynamics of such species.

The present review focuses on recent experimental and theoretical progress at the frontier of the field. It demonstrates how rotationally resolved photoelectron spectroscopy permits the investigation of molecular cations that are highly fluxional in their ground electronic state as a consequence of the JT or PJT effects. This article also introduces a group theoretical formalism 
that enables the assignment and interpretation of the experimental results. The experiments relied on the combination of pulsed-field-ionization zero-kinetic-energy (PFI-ZEKE) photoelectron spectroscopy $[20,21]$ with double-resonance excitation schemes involving mid-infrared, visible and vacuum-ultraviolet radiation. These developments were essential in assigning the complex spectra because, initially, no theoretical framework existed that could have guided the assignment procedure. Theoretically, a methodology has been developed to predict the rovibronic symmetry labels for the observed transitions of the molecules undergoing the JT effect. The method makes use of $a b$ initio quantum chemical calculations and of the complete nuclear permutation inversion (CNPI) or the molecular symmetry (MS) groups [22] that are adequate for highly fluxional systems. The $a b$ initio calculations are used to predict the minimum energy structures. Symmetry correlations between the CNPI group and the point group of the distorted minimum structures are used to predict all rovibronic levels, including their symmetry label.

For this review, we have chosen the methane cation as the first example of the JT effect in a triply degenerate electronic state characterized at the level of the rotational structure and the cyclopentadienyl cation as the prototype molecule to study the interplay of the PJT effect and antiaromaticity. The methane cation is one of the simplest systems subject to a $\mathrm{T} \otimes\left(\mathrm{e}+\mathrm{t}_{2}\right)$ JT effect and the cyclopentadienyl cation displays properties influenced by a complex interplay of PJT and JT effects. These two radical cations are of fundamental importance in chemistry and molecular physics but neither of them had been characterized in sufficient detail to obtain definitive structural and dynamical information prior to the work reviewed here. The reason for this situation lies in the difficulty of producing these charged species in sufficient quantities to allow for a spectroscopic investigation at high resolution.

The present article is structured as follows. Section 2 summarizes the theoretical methods used to describe the JT and PJT effects, with emphasis on group theory and its applications to the methane cation and the cyclopentadienyl cation. Section 3 describes the experimental methods, in particular the double-resonance excitation schemes used in the assignment of the rovibronic structure of photoelectron spectra. In Section 4, the general energy level structures of $\mathrm{CH}_{4}^{+}$and $\mathrm{C}_{5} \mathrm{H}_{5}^{+}$are described and interpreted. Section 5 is dedicated to two phenomena arising in some isotopically substituted molecules as a consequence of the JT effect: isomerism and chirality. Section 6 discusses the applicability of the group-theoretical method to other systems that are not subject to a JT effect. The last section consists of a short summary and an outlook. 


\section{Theoretical considerations}

\subsection{Vibronic coupling problems and molecular symmetry}

The Hamiltonian of a polyatomic molecule neglecting spins and molecular rotations can be written as

$$
\hat{H}=\hat{H}_{\mathrm{el} .}(\vec{r})+\hat{H}_{\text {nuc. }}(\vec{Q})+\hat{V}(\vec{r}, \vec{Q})
$$

where $\hat{H}_{\text {el. }}(\vec{r})$ is the electronic part including the kinetic energy of the electrons and their electrostatic interactions, $\hat{H}_{\text {nuc. }}(\vec{Q})$ is the kinetic energy operator of the nuclei and $\hat{V}(\vec{r}, \vec{Q})$ is the potential energy operator representing the interaction of the electrons and the nuclei and the internuclear repulsion. In standard treatments of the JT effect (e.g. $[1,2,17,23]), \hat{V}(\vec{r}, \vec{Q})$ is expanded in a Taylor series of small displacements of the nuclei about a reference geometry $\vec{Q}_{0}$ of highest symmetry

$$
\hat{V}(\vec{r}, \vec{Q})=\sum_{k=0}^{\infty} \frac{1}{k !} \sum_{i=1}^{3 N-6}\left(\frac{\partial^{k} \hat{V}}{\partial Q_{i}^{k}}\right)_{0} Q_{i}^{k},
$$

where $Q_{i}$ represents the symmetrized displacement coordinates (or vibrational modes). In Eq. (2) and throughout this article we neglect the contributions of terms containing mixed derivatives in two or more coordinates (so-called bilinear and higher-order coupling terms).

Retaining only the first term of the Taylor series $\hat{V}\left(\vec{r}, Q^{0}\right)$, the electronic part of the Schrödinger equation can be solved with fixed nuclei at the reference geometry

$$
\left[\hat{H}_{\mathrm{el} .}+\hat{V}\left(\vec{r}, Q^{0}\right)\right]\left|\phi_{i}(\vec{r})\right\rangle=\epsilon_{i}^{\prime}\left|\phi_{i}(\vec{r})\right\rangle
$$

giving a set of energies $\epsilon_{i}^{\prime}$ and so-called diabatic electronic wave functions $\left|\phi_{i}(\vec{r})\right\rangle$. The general solution of the Schrödinger equation for moving nuclei can be expanded in terms of the electronic functions

$$
\Psi(\vec{r}, \vec{Q})=\sum_{i} \chi_{i}(\vec{Q})\left|\phi_{i}(\vec{r})\right\rangle
$$

where the expansion coefficients $\chi_{i}(\vec{Q})$ are functions of the nuclear coordinates.

Expressing the Schrödinger equation corresponding to the Hamiltonian (1) in the basis of the diabatic electronic wave functions $\left|\phi_{i}(\vec{r})\right\rangle$ provides a system of coupled equations [1]

$$
\left[\hat{H}_{\text {nuc. }}+\epsilon_{i}(\vec{Q})^{\prime}+W_{i i}(\vec{Q})\right] \chi_{i}(\vec{Q})+\sum_{j \neq i} W_{i j} \chi_{i}(\vec{Q})=E \chi_{i}(\vec{Q})
$$

in which $W_{i j}$ are the elements of the vibronic interaction matrix

$$
W(\vec{r}, \vec{Q})=V(\vec{r}, \vec{Q})-V\left(\vec{r}, Q^{0}\right)
$$


and $\epsilon_{i}(\vec{Q})^{\prime}$ is the eigenenergy of the diabatic electronic wave function defined in Eq. (3) at the arbitrary geometry $\vec{Q}$.

If the vibronic interactions can be neglected, the coupled equations (5) decompose into a set of simple equations that can be solved independently for each electronic state, which is known as the Born-Oppenheimer approximation. In the opposite case, a separation is not possible and the coupled equations (5) must be solved together.

The solution of a particular vibronic coupling problem is considerably simplified by exploiting the molecular symmetry. The operators in Eq. (3) and, in particular, the potential energy operator in Eq. (2) are invariant under all operations of the complete nuclear permutation inversion (CNPI) group of the molecule [22]. A nonvanishing contribution to vibronic coupling between the electronic states of irreducible representations $\Gamma_{\mathrm{A}}$ and $\Gamma_{\mathrm{B}}$ only exists for a term of order $k$ in Eq. (2) if

$$
\Gamma_{\mathrm{A}} \otimes \Gamma_{\mathrm{B}} \supseteq\left[\Gamma_{\nu}\right]^{k}
$$

where $\left[\Gamma_{\nu}\right]^{k}$ is the symmetric $k^{\text {th }}$ power of the irreducible representation of the vibrational mode $\nu$. The corresponding vibration is then called "active". Vibronic coupling between the different components of a degenerate electronic state of irreducible representation $\Gamma_{\text {el,deg. }}$, which is known as the Jahn-Teller effect, is allowed along mode $\nu$ if

$$
\left[\Gamma_{\text {el,deg. }}\right]^{2} \supseteq\left[\Gamma_{\nu}\right]^{k}
$$

and the coupling between a degenerate state $\left(\Gamma_{\text {el,deg. }}\right)$ and a non-degenerate state $\left(\Gamma_{\text {el,nondeg. }}\right)$, the so-called Pseudo-Jahn-Teller effect, can only occur along mode $\nu$ if

$$
\Gamma_{\text {el,deg. }} \otimes \Gamma_{\text {el,nondeg. }} \supseteq\left[\Gamma_{\nu}\right]^{k}
$$

After identification of the JT and PJT active modes, the vibronic Hamiltonian is set up in the basis of diabatic electronic states defined in Eq. (3). The active modes are designated by their irreducible representation $\Gamma_{\nu}$ which has several components $\gamma$ if the representation is degenerate. The kinetic energy term of the nuclear Hamiltonian can be expressed as

$$
\hat{H}_{\text {kin }}=\sum_{\nu_{\gamma}}-\frac{\hbar^{2}}{2 \mu_{\nu}} \frac{\partial^{2}}{\partial Q_{\nu_{\gamma}}^{2}} \mathbf{1}
$$

where $\mu_{\nu}$ is the reduced mass of the vibrational mode. The potential energy term, truncated to second order, is

$$
V(\vec{Q})=\sum_{\nu_{\gamma}}\left(\frac{1}{2} \mu_{\nu} \omega_{\nu}^{2} Q_{\nu_{\gamma}}^{2} \mathbf{1}+V_{\nu} Q_{\nu_{\gamma}} \mathbf{C}_{\nu_{\gamma}}+K_{\nu} Q_{\nu_{\gamma}}^{2} \mathbf{1}\right)
$$


where $V_{\nu}$ and $K_{\nu}$ are the linear and quadratic coupling constants and $\omega_{\nu}$ is the vibrational frequency of the vibrational mode $\nu . \mathbf{C}_{\nu_{\gamma}}$ is a matrix of Clebsch-Gordan coefficients that can be found in tables $[24,25]$.

Adiabatic potential energy surfaces $E(\vec{Q})$ and electronic state vectors $|a(\vec{Q})\rangle$ are obtained by solving

$$
V(\vec{Q})|a(\vec{Q})\rangle=E(\vec{Q})|a(\vec{Q})\rangle
$$

These quantities provide a simple picture of the vibronic coupling problem that reveals its essential aspects of symmetry and topology as will be shown below.

The comparison with observed energy levels requires solving the coupled electronic-vibrational problem, which is in general very difficult. The traditional approach consists in representing the problem in a direct product basis of diabatic electronic functions and harmonic oscillator functions of the appropriate degeneracy and subsequently diagonalizing the matrices $[5,15,17]$. The vibrational basis is increased until convergence is reached. The size of the matrices grows very quickly with the magnitude of the Jahn-Teller stabilization energy and the number of involved vibrational modes. In spite of efficient iterative diagonalization algorithms (e.g., the Lanczos algorithm [26]), only a small number of modes can be treated simultaneously if the stabilization energy is large. This procedure is applied below to the analysis of the spectra of the cyclopentadienyl cation. The lowest singlet state is subject to a PJT stabilization of $\sim 5000 \mathrm{~cm}^{-1}$ involving four doubly degenerate modes. In the case of the methane cation, which undergoes a JT stabilization of $\sim 12000 \mathrm{~cm}^{-1}$ mediated by two triply and one doubly degenerate modes, the solution of the coupled problem is a tremendous task, and we therefore opted for a simplified effective Hamiltonian approach which exploits the molecular symmetry.

\subsection{The role of $a b$ initio calculations in the elucidation of JT problems}

Traditional ab initio quantum chemical calculations are performed at a frozen configuration of the nuclei according to Eq. (3) in the realm of the Born-Oppenheimer (BO) approximation. Since the JT effect results from the coupling between nuclear and electronic degrees of freedom, which implies a failure of the BO approximation, it is justified to wonder about the utility of $a b$ initio calculations according to Eq. (3) in understanding JT problems.

$A b$ initio calculations are capable of predicting the minimum energy geometry and the amount of electronic potential energy by which a molecule is stabilized compared to the geometry of highest symmetry where two or more electronic states are degenerate (the so-called JT stabilization energy). These parameters, as well as the intersections of electronic potential 
surfaces are nowadays accessible by specialized methods of $a b$ initio calculation and the JT coupling parameters can be efficiently predicted (see, e.g., [27]). These data are essential parameters to describe the JT effect in a molecule, but are in general insufficient to predict the coupled nuclear and electronic dynamics and the rovibronic energy level structure of a molecule. Indeed, in molecules with a high permutational symmetry, the JT effect leads to several equivalent minima on the potential energy surface. If these minima are connected by low-energy barriers, large amplitude motions take place giving rise to complex quantum level structures. In this article, we illustrate the use of $a b$ initio calculations to predict the molecular geometry at the minima of the potential energy surface and the use of group theory to predict the resulting rovibronic quantum states.

The most accurate solution of the coupled electronic-vibrational problem is given by the formalism outlined in Section 2.1. It provides the complete solution of the vibronic problem for arbitrary energies, including regions where electronic surfaces intersect and the BO approximation breaks down. In cases like that of the methane cation which has a JT stabilization energy of $\sim 1.5 \mathrm{eV}$, this procedure is too demanding and a simpler solution is needed. Indeed, when the JT stabilization energy is large, the BO approximation provides an accurate description of the nuclear dynamics in the region of the potential energy minima. However, one essential aspect needs consideration. The adiabatic electronic wave function changes its character from one equivalent minimum to another. This property can be described by a geometric (or topological) phase [28] which affects the quantum level structure of JT systems. In the case of the E $\otimes \mathrm{e}$ JT effect, the geometric phase reverses the energetic ordering of vibronic levels from A below $\mathrm{E}$ to $\mathrm{E}$ below $\mathrm{A}[9]$. In the $\mathrm{T}_{2} \otimes\left(\mathrm{e}+\mathrm{t}_{2}\right)$ case, it even changes the symmetry of vibronic levels from $\mathrm{A}$, $\mathrm{F}_{2}$, E to $\mathrm{F}_{2}, \mathrm{~F}_{1}{ }^{a}[29]$.

\subsection{Jahn-Teller effects in diradicals of configuration $\mathrm{e}^{2}$}

Diradicals possessing two electrons in a degenerate orbital form an important class of molecules which encompasses the so-called "antiaromatic" molecules, the simplest of which are the cyclopropenyl anion, cyclobutadiene and the cyclopentadienyl cation. In the following, we briefly formulate the vibronic coupling theory describing these systems, emphasizing the simplifications arising from symmetry considerations. The left-hand side of Fig. 1 displays the energetic or-

\footnotetext{
${ }^{a}$ The commonly used label for the JTE still employs the letters $\mathrm{T}$ or $\mathrm{t}$ to designate triply degenerate electronic states and vibrational modes, respectively, rather than the recommended $\mathrm{F}$ and $\mathrm{f}$ labels used in the remainder of this article.
} 
dering of the $\mathrm{a}_{2}^{\prime \prime}$, $\mathrm{e}_{1}^{\prime \prime}$ and $\mathrm{e}_{2}^{\prime \prime}$ molecular orbitals of $\mathrm{C}_{5} \mathrm{H}_{5}^{+}$in the form of a Frost-Musulin diagram. In the $\mathrm{D}_{n \mathrm{~h}}(\mathrm{M})$ symmetry groups with $n$ odd, the ground configuration $\left(\mathrm{a}_{2}^{\prime \prime}\right)^{2}\left(\mathrm{e}_{1}^{\prime \prime}\right)^{2}$ gives rise to the three states ${ }^{3} \mathrm{~A}_{2}^{\prime},{ }^{1} \mathrm{E}_{2}^{\prime}$ and ${ }^{1} \mathrm{~A}_{1}^{\prime}$ displayed on the right-hand side of Fig. 1. The Hartree-Fock energies of these three states are $2 h+J_{23}-K_{23}, 2 h+J_{23}+K_{23}$ and $2 h+J_{22}+K_{23}$, respectively, where $h, J_{i j}$ and $K_{i j}$ represent the one-electron orbital energy, the Coulomb and the exchange integrals, respectively, and the indices designate the $\pi$ molecular orbitals in order of increasing energy [30] (by symmetry $J_{22}-J_{23}=2 K_{23}$ ).

Neglecting spin-orbit interactions and coupling with higher-lying triplet states, the ${ }^{3} \mathrm{~A}_{2}^{\prime}$ ground electronic state of $\mathrm{C}_{5} \mathrm{H}_{5}^{+}$does not undergo vibronic coupling. The lowest-lying singlet state ${ }^{1} \mathrm{E}_{2}^{\prime}$ is degenerate and, in this state, the $e_{1}^{\prime}$ modes induce a linear JT effect and the $e_{2}^{\prime}$ and $e_{2}^{\prime \prime}$ modes induce a quadratic JT effect. The $e_{2}^{\prime}$ modes also induce a linear PJT coupling between the ${ }^{1} \mathrm{E}_{2}^{\prime}$ and the ${ }^{1} \mathrm{~A}_{1}^{\prime}$ states. These results and those for higher-order vibronic interactions are summarized in Table I.

The coupled Hamiltonian is most conveniently set up in a diabatic electronic basis. Using complex basis functions for the E state and retaining only linear coupling terms, the Hamiltonian matrix $\mathrm{H}$ can be written as [31]

$$
H=H^{\mathrm{PJT}}+H^{\mathrm{JT}},
$$

where

$$
H^{\mathrm{PJT}}=h_{0}^{\mathrm{PJT}} \mathbf{1}+\left(\begin{array}{ccc}
E_{\mathrm{E}} & 0 & \sum_{j} \lambda_{j} r_{j} e^{i \phi_{j}} \\
0 & E_{\mathrm{E}} & \sum_{j} \lambda_{j} r_{j} e^{-i \phi_{j}} \\
\text { H.c. } & \text { H.c. } & E_{\mathrm{A}}
\end{array}\right)
$$

and

$$
H^{\mathrm{JT}}=h_{0}^{\mathrm{JT}} \mathbf{1}+\left(\begin{array}{ccc}
0 & \sum_{n} g_{n} \rho_{n} e^{-i \theta_{n}} & 0 \\
H . c . & 0 & 0 \\
0 & 0 & 0
\end{array}\right) .
$$

The degenerate vibrational modes mediating the vibronic coupling are described by cylindrical mass-weighted dimensionless normal coordinates $r_{j}, \phi_{j}$, and $\rho_{n}, \theta_{n}$, respectively, which are related to the Cartesian coordinates $x_{j}$ and $y_{j}\left(x_{n}\right.$ and $\left.y_{n}\right)$ as indicated at the bottom of Figs. 2 and 3. The first and second terms in Eq. (13) represent the PJT and JT interactions, respectively (H.c. means "Hermitian conjugate"), with linear coupling constants $\lambda_{j}$ and $g_{n}$, respectively. The indices $j$ and $n$ label the PJT and JT active modes, respectively. The harmonic oscillator terms $h_{0}^{\mathrm{PJT}}$ and $h_{0}^{\mathrm{JT}}$ in Eqs. (14) and (15) take the form

$$
h_{0}^{\mathrm{PJT}}=\sum_{j} \frac{\omega_{j}}{2}\left(-\frac{1}{r_{j}} \frac{\partial}{\partial r_{j}} r_{j} \frac{\partial}{\partial r_{j}}-\frac{1}{r_{j}^{2}} \frac{\partial^{2}}{\partial \phi_{j}^{2}}+r_{j}^{2}\right)
$$


and

$$
h_{0}^{\mathrm{JT}}=\sum_{n} \frac{\omega_{n}}{2}\left(-\frac{1}{\rho_{n}} \frac{\partial}{\partial \rho_{n}} \rho_{n} \frac{\partial}{\partial \rho_{n}}-\frac{1}{\rho_{n}^{2}} \frac{\partial^{2}}{\partial \theta_{n}^{2}}+\rho_{n}^{2}\right)
$$

respectively.

The adiabatic potential energy surfaces have a simple appearance when only either JT or PJT displacements are considered. In the case of a single JT-active mode with cylindrical coordinates $(\rho, \theta)$ a characteristic potential is obtained for the E state

$$
V_{\mathrm{E}^{ \pm}}(\rho, \theta)=V_{\mathrm{E}^{ \pm}}(\rho)=V_{\mathrm{E}}(0)+\frac{\omega_{\mathrm{JT}}}{2} \rho^{2} \pm g \rho,
$$

as represented in Fig. 2.

For a single PJT-active mode with cylindrical coordinates $(r, \phi)$, two of the surfaces repel each other whereas the third remains unchanged. Assuming identical vibrational frequencies in the $\mathrm{A}$ and $\mathrm{E}$ states, the following potential surfaces are obtained

$$
\begin{aligned}
V_{A}(r, \phi)=V_{A}(r) & =\frac{V_{\mathrm{E}}(0)+V_{\mathrm{A}}(0)}{2}+\frac{\omega_{\mathrm{PJT}}}{2} r^{2}+\sqrt{\left[\frac{V_{\mathrm{A}}(0)-V_{\mathrm{E}}(0)}{2}\right]^{2}+2 \lambda^{2} r^{2}} \\
V_{E_{y}}(r, \phi)=V_{E_{y}}(r) & =V_{\mathrm{E}}(0)+\frac{\omega_{\mathrm{PJT}}}{2} r^{2} \\
V_{E_{x}}(r, \phi)=V_{E_{x}}(r) & =\frac{V_{\mathrm{E}}(0)+V_{\mathrm{A}}(0)}{2}+\frac{\omega_{\mathrm{PJT}}}{2} r^{2}-\sqrt{\left[\frac{V_{\mathrm{A}}(0)-V_{\mathrm{E}}(0)}{2}\right]^{2}+2 \lambda^{2} r^{2}},
\end{aligned}
$$

as represented in Fig. 3.

JT and PJT interactions both lift the electronic degeneracy in the ${ }^{1} \mathrm{E}_{2}^{\prime}$ state but the effects are qualitatively different as can be seen in Fig. 4. Both $\mathrm{e}_{1}^{\prime}$ and $\mathrm{e}_{2}^{\prime}$ modes have two components $\mathrm{e}_{\mathrm{x}}^{\prime}$ and $\mathrm{e}_{\mathrm{y}}^{\prime}$. A distortion along an $\mathrm{e}_{\mathrm{x}}^{\prime}$ component conserves $\mathrm{C}_{2 \mathrm{v}}$ symmetry, whereas a distortion along an $\mathrm{e}_{\mathrm{y}}^{\prime}$ component lowers the symmetry to $\mathrm{C}_{\mathrm{s}}$. A distortion from $\mathrm{D}_{5 \mathrm{~h}}$ to $\mathrm{C}_{2 \mathrm{v}}$ symmetry splits the ${ }^{1} \mathrm{E}_{2}^{\prime}$ state into two components of symmetry ${ }^{1} \mathrm{~A}_{1}$ and ${ }^{1} \mathrm{~B}_{2}$ (full and dashed lines, respectively). In the case of a linear JT effect, the potential energy surfaces have a nonzero slope at the point of $\mathrm{D}_{5 \mathrm{~h}}$ symmetry and, consequently, their ordering is reversed when the sign of the distortion coordinate is changed. In the case of a quadratic JT or a PJT effect, the slope of the adiabatic electronic surfaces vanishes at the point of highest symmetry and the ordering of the two components is independent of the sign of the distortion coordinate. In the present case, the lower component is totally symmetric $\left({ }^{1} \mathrm{~A}_{1}\right.$ in $\left.\mathrm{C}_{2 \mathrm{v}}\right)$ whereas the upper component has symmetry ${ }^{1} \mathrm{~B}_{2}$ in $\mathrm{C}_{2 \mathrm{v}}$.

The linear JT effect in an electronic state having two electrons in two degenerate orbitals (configuration $\mathrm{e}^{2}$ ) vanishes if configuration interaction is neglected [32] and the coupling constants of the three $\mathrm{e}_{1}^{\prime}$ modes in the ${ }^{1} \mathrm{E}_{2}^{\prime}$ state are indeed found to be very small $[33,34]$. The 
linear PJT effect in the four modes of symmetry $\mathrm{e}_{2}^{\prime}$ on the contrary is very strong and leads to a deep minimum energy trough on the lowest potential energy surface as shown in Fig. 3. The distortion of the molecule changes continuously as one moves along the minimum of the surface as shown in Fig. 5 a). Fig. 5 b) illustrates the geometric and electronic structure of the molecule if the distortion follows an $\mathrm{e}_{2 \mathrm{x}}^{\prime}$ distortion from the reference geometry to the two minima. The remarkable property is the absence of quadratic PJT coupling in the $\mathrm{e}_{2}^{\prime}$ modes which is imposed by the molecular symmetry (see Table I) and implies that pseudorotation along the circular minimum energy path in Fig. 3 (which corresponds to the circle depicted in Fig. 5a) is unhindered. The vibronic singlet ground-state wave function of the cyclopentadienyl cation is thus completely delocalized over the pseudorotation path.

\subsection{The Jahn-Teller effect in a triply degenerate electronic state}

Threefold degenerate electronic states are common in highly symmetric molecules with unpaired electrons like $\mathrm{CH}_{4}^{+}$or $\mathrm{P}_{4}^{+}$and especially also in transition metal complexes. The JT problem resulting from the configuration $\mathrm{t}^{1}$ (or $\mathrm{t}^{5}$ ) is very rich and has been studied theoretically in Ref. [24]. In a triply degenerate electronic state, the twofold and threefold degenerate vibrational modes (e and f) are JT active. Distortion along a mode of vibrational symmetry e leads to a minimum energy geometry of tetragonal symmetry, whereas distortion along an f mode results in a trigonal minimum geometry. If JT activity is limited to the linear term, these are the only possible minima, even when both modes are simultaneously active.

Only when quadratic JT activity is permitted does a new kind of minima appear, which is of lower symmetry. This case, which applies to the methane cation, is illustrated in Figs. 6 and 7. The tetrahedral configuration corresponds to a triply degenerate conical intersection. If the molecule is distorted along the two vibrational modes of symmetry $f_{2}$ it can reach either the structure $\mathrm{C}_{3 \mathrm{v}}(\mathrm{II})$ or the structure $\mathrm{C}_{3 \mathrm{v}}(\mathrm{I})$ depicted in Fig. 7. The former possesses one short and three long $\mathrm{C}-\mathrm{H}$ bonds, and has a doubly degenerate ground state $\left({ }^{2} \mathrm{E}\right.$ in $\left.\mathrm{C}_{3 \mathrm{v}}(\mathrm{M})\right)$ corresponding to a twofold conical intersection and a nondegenerate first excited state $\left({ }^{2} A_{1}\right.$ in $\left.C_{3 v}(M)\right)$. The latter possesses one long and three short $\mathrm{C}-\mathrm{H}$ bonds and has a nondegenerate ground electronic state $\left({ }^{2} \mathrm{~A}_{1}\right.$ in $\left.\mathrm{C}_{3 \mathrm{v}}(\mathrm{M})\right)$ and a doubly degenerate first excited electronic state $\left({ }^{2} \mathrm{E}\right.$ in $\left.\mathrm{C}_{3 \mathrm{v}}(\mathrm{M})\right)$. Further distortions along the e modes lead to the $\mathrm{C}_{2 \mathrm{v}}$ minimum energy structure depicted in Fig. 6 and which are characterized by two long C-H bonds separated by a small angle $\left(\sim 60^{\circ}\right)$ and two short $\mathrm{C}-\mathrm{H}$ bonds separated by a large angle $\left(\sim 127^{\circ}\right)$.

The total stabilization energy amounts to about $12000 \mathrm{~cm}^{-1}$ [35-37]. The number of equiv- 
alent structures of a given symmetry is determined by the ratio of the orders of the CNPI group of the molecule and the point group of the structure. The CNPI of $\mathrm{CH}_{4}^{+}$has order 48 and therefore there are 8 equivalent structures of each of the two $\mathrm{C}_{3 \mathrm{v}}$ geometries displayed in Fig. 7, 6 of $\mathrm{D}_{2 \mathrm{~d}}$ geometry and 12 of $\mathrm{C}_{2 \mathrm{v}}$ geometry. The 12 possible $\mathrm{C}_{2 \mathrm{v}}$ structures fall in two sets of six structures, each of which are mirror images of each other [35]. One of these sets is represented in Fig. 8 along with the adiabatic electronic function corresponding to the local minimum expressed as linear combination of the three diabatic electronic functions $|\eta\rangle,|\xi\rangle$, and $|\zeta\rangle$. The very large stabilization energy and the eight-dimensional space of JT active vibrations render a complete vibronic calculation as described in the previous section intractable. Simplifications arise, however, from the consideration of the properties of the potential energy surfaces. Equivalent minima are separated by relatively low barriers that permit the interconversions of neighboring structures by tunneling. In the following, the tunneling formalism is derived which is then used to predict the rovibronic energy levels including their symmetry.

The barrier height separating two enantiomeric $\mathrm{C}_{2 \mathrm{v}}$ structures has been calculated to be $5100 \mathrm{~cm}^{-1}$ at the UMP2/cc-pVDZ level of ab initio theory $\left(4700 \mathrm{~cm}^{-1}\right.$ including zero-point corrections). The barrier separating two $\mathrm{C}_{2 \mathrm{v}}$ minima in Fig. 8 amounts to only $1010 \mathrm{~cm}^{-1}$ at the same level of theory and only $410 \mathrm{~cm}^{-1}$ after zero-point correction (although a harmonic zero-point correction is questionable for a flat potential energy surface). The motion connecting two enantiomeric structures corresponds to the stereomutation of the methane cation $[38,39]$. The resulting splittings are estimated to be less than $10^{-7} \mathrm{~cm}^{-1}$ and cannot be observed at our experimental resolution of $0.2 \mathrm{~cm}^{-1}$. The motion connecting equivalent minima of a given set of six structures corresponds to a cyclic permutation of three hydrogen atoms also known as pseudorotation. The low barrier for this process implies that it must be observable at our resolution. Inspection of Fig. 8 reveals that the feasible permutation-inversion operations are of the type E, (123), (12)(34), (12)*,$(1234)^{*}$ and form a group that is known as $\mathrm{T}_{\mathrm{d}}(\mathrm{M})$ in the literature [22]. The molecular symmetry group used in Ref. [40] contains operations of the type E, (123), (12)(34), (12), (1234), which is incorrect because the last two operations involve an odd number of permutations and thus interconvert enantiomers.

Tunneling between the six structures depicted in Fig. 8 is conveniently described in a matrix representation using direct product basis states

$$
\phi_{n}=|n\rangle\left|\chi_{n}\right\rangle,
$$

where $|n\rangle$ is the adiabatic electronic state vector in the $n^{\text {th }}$ potential well (given below each 
structure in Fig. 8) and $\chi_{n}$ represents the vibrational ground-state function that is sharply localized in the vicinity of the $n^{\text {th }}$ potential well. Neglecting the overlap between the vibrational basis functions and defining $\sigma=1 / 2\left\langle\chi_{1}\left|\hat{H}_{\text {vib }}\right| \chi_{2}\right\rangle$ and $\left\langle\chi_{1}\left|\hat{H}_{\text {vib }}\right| \chi_{1}\right\rangle=0$, where $\hat{H}$ is the vibrational operator for the lowest potential energy surface, the effective Hamiltonian matrix can be expressed as

$$
\mathbf{H}_{\mathrm{vib}}=\left(\begin{array}{cccccc}
0 & \sigma & -\sigma & \sigma & -\sigma & 0 \\
\sigma & 0 & \sigma & 0 & -\sigma & \sigma \\
-\sigma & \sigma & 0 & -\sigma & 0 & \sigma \\
\sigma & 0 & -\sigma & 0 & \sigma & \sigma \\
-\sigma & -\sigma & 0 & \sigma & 0 & \sigma \\
0 & \sigma & \sigma & \sigma & \sigma & 0
\end{array}\right) .
$$

The tunneling eigenstates of $\mathrm{CH}_{4}^{+}$are obtained by diagonalizing $\mathrm{H}_{\text {vib }}$ and form two sets of triply degenerate levels with eigenvalues

$$
\begin{aligned}
& E_{\mathrm{F}_{2}}=+2 \sigma \\
& E_{\mathrm{F}_{1}}=-2 \sigma
\end{aligned}
$$

separated by the tunneling splitting $\delta=4|\sigma|$ ( $\sigma$ is negative). The subscript indicates the symmetry of the vibronic wavefunction in the $\mathrm{T}_{\mathrm{d}}(\mathrm{M})$ group. The high permutational symmetry of $\mathrm{CH}_{4}^{+}$is responsible for the equality of all matrix elements $\left\langle\chi_{i}\left|\hat{H}_{\mathrm{vib}}\right| \chi_{i}\right\rangle$ and all tunneling elements $\left\langle\chi_{i}\left|\hat{H}_{\mathrm{vib}}\right| \chi_{j}\right\rangle$. Each component of the triply degenerate vibronic wavefunctions has amplitudes in four of the minima [41].

The vibronic ground state wavefunctions of both the cyclopentadienyl cation and the methane cation are delocalized over areas of the potential energy surfaces that correspond to different but equivalent molecular structures. Group theoretical methods can be used to predict the symmetries of the vibronic wavefunctions. First, the barriers separating equivalent minima are assumed to be infinitely high and the symmetry of the vibronic wave function is determined locally. Since the molecule is rigid and in its ground vibrational state, it must be classified in the point group of the distorted structure and the vibronic symmetry is the same as its electronic symmetry. This is $\mathrm{B}_{2}$ in the case of $\mathrm{CH}_{4}^{+}$, as can be seen from the structure of the singly occupied molecular orbital displayed in Fig. 6. In defining the symmetry, we have used the $\mathrm{C}_{2 \mathrm{v}}$ character table given in Ref. [42]. If tunneling is taken into account, the vibronic symmetries must be classified in the CNPI group and are obtained by correlating the irreducible representations of $C_{2 v}$ to $T_{d}(M)$. $\mathrm{B}_{2}$ becomes $\mathrm{F}_{2} \oplus \mathrm{F}_{1}$ which is in agreement with the result of the tunneling matrix Eq. (21). 
This procedure can also be used to derive the vibronic symmetries of the partially deuterated methane cations, as will be explained in Subsection 5.1.

\section{$3 \quad$ Experimental methods}

The investigation of molecular ions by direct spectroscopy is a challenging task. Indeed, the repulsion forces between cations in a gas sample limit their concentration to below $10^{10} \mathrm{~cm}^{-3}$, and cause undesired Doppler broadenings of the transitions. Moreover, the techniques used to generate the cations (electron impact ionization, discharges, etc.) are neither chemically nor state selective and the extraction of relevant spectral features from experimental data represents a real challenge. In photoelectron spectroscopy, the cation energy level structure is measured from the neutral ground state of the molecule. Efficient and chemically selective routes exist to produce neutral species and supersonic expansions can lead to the population of only the lowest quantum states. Moreover, the photoionizing transitions to specific cationic states can be detected with almost $100 \%$ efficiency by measuring electrons.

In PFI-ZEKE photoelectron spectroscopy, electrons produced by the electric field ionization of very high Rydberg states (principal quantum number $n \approx 300$ ) are measured as a function of the wave number of a tunable light source $[20,21,43,44]$. The lines in a PFI-ZEKE photoelectron spectrum are located just below each ionization threshold and thus directly map out the relative positions of the ionic energy levels. To obtain the field-free ionization thresholds, a field correction has to be applied [45].

The experimental setup has been described previously [37]. Briefly, the neutral molecules in a supersonic expansion are photoexcited inside a stack of resistively coupled extraction plates. For photoionization measurements, the produced ions are extracted using a pulsed voltage toward a multichannel plate detector where they are detected mass-selectively. For PFI-ZEKE experiments, an electric field pulse sequence consisting typically of a discrimination pulse of $+0.05 \mathrm{~V} / \mathrm{cm}$ and a detection pulse of $0.2 \mathrm{~V} / \mathrm{cm}$ delayed by $3 \mu$ s with respect to photoexcitation was used, resulting in a resolution of $0.3 \mathrm{~cm}^{-1}$.

In order to simplify the PFI-ZEKE photoelectron spectra and to provide an experimental assignment of the rovibronic symmetry of the observed cationic levels, various double-resonance excitation schemes were used. The resonance-enhanced two-photon excitation/ ionization experiments were carried out using two tunable laser sources. The laser beams crossed the molecular beam at right angles in a counterpropagating arrangement. By scanning the first laser at a 
constant wave number of the second laser, spectra of the intermediate state were recorded. PFI-ZEKE photoelectron spectra were measured from selected vibrational levels of the intermediate level by fixing the wave number of the first laser at the appropriate spectral position and scanning the wave number of the second laser.

Two schemes were used to assign the nuclear spin species and the rovibronic symmetry of the ionic levels experimentally. The first technique, that we call "ZEKE-dip" spectroscopy (by analogy with ion-dip spectroscopy [46]) was applied to assign the rovibronic symmetries of the levels of the methane cation. It consists in holding the VUV frequency at the position of a given line in the single-photon PFI-ZEKE photoelectron spectrum, and monitoring the depletion of the photoelectron signal at specific frequencies of an IR laser that is resonant with rovibrational transitions in the $\nu_{3}$ or $2 \nu_{3}$ bands of neutral methane. A depletion of up to $50 \%$ of the original signal indicated that the IR and the VUV photoelectronic transitions had a common rovibrational lower level that is easily identifiable by comparison with the well-known IR spectrum of $\mathrm{CH}_{4}$ [47]. In the second technique, the IR frequency was held at the position of specific lines of the IR spectrum, and the VUV frequency was scanned across the ionization thresholds. With these two methods, nuclear spin symmetries could be assigned to most lines in the PFI-ZEKE photoelectron spectrum (the nuclear spin symmetry is assumed to be conserved during photoionization).

The investigation of the cyclopentadienyl cation required the production of the cyclopentadienyl radical under conditions that are compatible with PFI-ZEKE photoelectron spectroscopy. Such a source has been developed recently [48]. The cyclopentadienyl radicals were produced by photolysis of cyclopentadiene with the $248 \mathrm{~nm}$ output of a $\mathrm{KrF}$ excimer laser (Lambda Physik, CompEx) in a quartz capillary mounted at the end of a pulsed nozzle [34,49]. The cyclopentadienyl radicals were cooled in the pulsed supersonic expansion into vacuum to rotational temperatures around $8 \mathrm{~K}$. The supersonic beam was skimmed and then intersected by the VUV beam at right angles in the photoionization region. Cyclopentadiene was produced from dicyclopentadiene (Fluka) through distillation and stored at $-78{ }^{\circ} \mathrm{C}$ until used. It was introduced in a stream of neat helium into the nozzle reservoir at a stagnation pressure of 5 bar.

\section{Comparison with experimental data}

The theoretical considerations presented in Section 2 have explained how the JT and PJT effects lower the potential energy for nuclear configurations of reduced symmetry. In $\mathrm{CH}_{4}^{+}$and $\mathrm{C}_{5} \mathrm{H}_{5}^{+}$ 
and their deuterated isotopomers, different equivalent minima appear with small to vanishing barriers between them. Consequently, a molecule subject to a JT effect often samples several equivalent distorted geometries which translates into complex spectra. This section illustrates the spectroscopic signatures of this fluxionality caused by the JT effect. The spectroscopic experiments serve the purpose of determining the quantum level structure of the molecular cation including nuclear spin symmetry which provides information on its structure and the dynamics.

\subsection{Cyclopentadienyl cation}

Prior to our investigations by PFI-ZEKE photoelectron spectroscopy $[34,49]$ the cyclopentadienyl cation had only been studied in the condensed phase by electron paramagnetic resonance spectroscopy [50]. The excitation schemes used in our experiments are represented in Fig. 9. Direct one-photon excitation in the VUV from the $\tilde{\mathrm{X}}^{2} \mathrm{E}_{1}^{\prime \prime}$ ground state of the cyclopentadienyl radical enabled us to record the PFI-ZEKE photoelectron spectrum of the ground state of the cyclopentadienyl cation [49]. The spectrum is dominated by the transition to the vibrational ground state of the cation. Since the Jahn-Teller distortion of the electronic ${ }^{2} \mathrm{E}_{1}^{\prime \prime}$ ground state of the radical is relatively weak, this observation indicates that the $\tilde{\mathrm{X}}^{+}{ }^{3} \mathrm{~A}_{2}^{\prime}$ ground state of the cyclopentadienyl cation is insignificantly distorted away from the $\mathrm{D}_{5 \mathrm{~h}}$ structure. A second excitation scheme was used to characterize higher-lying electronic states. $\left(1+1^{\prime}\right)$-two-photon resonant excitation via different vibrational levels of the electronically excited $\tilde{\mathrm{A}}^{2} \mathrm{~A}_{2}^{\prime \prime}$ state of the neutral radical gave access to a dense progression of vibrational levels beginning about 1500 $\mathrm{cm}^{-1}$ above the measured origin of the ${ }^{3} \mathrm{~A}_{2}^{\prime}$ state [49]. The PFI-ZEKE photoelectron spectra recorded following excitation via the vibrational ground state of the ${ }^{2} \mathrm{~A}_{2}^{\prime \prime}$ state of the neutral radicals $\mathrm{C}_{5} \mathrm{H}_{5}$ and $\mathrm{C}_{5} \mathrm{D}_{5}$ are displayed in Figs. 10a and 10b, respectively. The vertical arrows on the low wave number side of Fig. 10 correspond to the positions where the origin of the ${ }^{3} \mathrm{~A}_{2}^{\prime}$ has been observed in the single-photon PFI-ZEKE experiments [49]. The origins of the $\tilde{\mathrm{X}}^{+} \leftarrow \tilde{\mathrm{X}}$ photoelectron band systems are not observed in the two-photon experiments but several vibrationally excited levels of the $\tilde{\mathrm{X}^{+}{ }^{3} \mathrm{~A}_{2}^{\prime}}$ electronic state are observed: $14^{2}, 2^{1}, 13^{1}, 14^{1}, 10^{1}$ and $13^{2}$ in $\mathrm{C}_{5} \mathrm{H}_{5}^{+}$and $2^{1}, 8^{2}$ in $\mathrm{C}_{5} \mathrm{D}_{5}^{+}$. At a total wave number of approximately $69500 \mathrm{~cm}^{-1}$, the origin of the $\tilde{\mathrm{a}}^{+1} \mathrm{E}_{2}^{\prime} \leftarrow \tilde{\mathrm{X}}$ photoelectron band system is observed in both molecules as a rather weak line (labeled " $\mathrm{j}=0$ ") immediately followed by a much more intense line labeled $\mathrm{j}=1$ and further weak lines. A second similar group of lines is observed at higher wave numbers around $70400 \mathrm{~cm}^{-1}$. The lines of these two progressions are assigned in terms of the quantum number $j$ that stands 
for the vibronic angular momentum [34]. The upper progression is attributed to the excited vibrational level with one quantum of excitation in the dimension perpendicular to the tangent of the trough. The separation between the two lowest lines in the progressions amounts to only 71 $\mathrm{cm}^{-1}$ in $\mathrm{C}_{5} \mathrm{H}_{5}^{+}$and $70 \mathrm{~cm}^{-1}$ in $\mathrm{C}_{5} \mathrm{D}_{5}^{+}$which is characteristic of a very strong Pseudo-Jahn-Teller distortion (see Fig. 3) and the associated free pseudorotation of the nuclear framework depicted in Fig. 5. The motion along the trough in the lowest potential surface of Fig. 3 is indeed expected to be unhindered as explained in Section 2.3. From the experimental observations one can conclude that the quantized states corresponding to this motion have an energy proportional to $j^{2}$ with integer values of the vibronic angular momentum quantum numbers $j=0,1,2 \ldots$ In the case of a strong JT (as opposed to a PJT) distortion, the energy would also be proportional to $j^{2}$ but with half-integer values of $j(1 / 2,3 / 2 \ldots)$ (see also Refs. [9-11]). The assignment of the spectra displayed in Fig. 10 relied on ab initio quantum chemical calculations, vibronic coupling calculations and rotationally resolved measurements [34].

The unexpected intensity distribution originates from the fact that the photoionizing transition from the ${ }^{2} \mathrm{~A}_{2}^{\prime \prime}$ state to the ${ }^{1} \mathrm{E}_{2}^{\prime}$ state of the cation is forbidden, as it is formally a two-electron transition (see Fig. 9). The allowed photoionizing transitions from the ${ }^{2} \mathrm{~A}_{2}^{\prime \prime}$ state lead to the formation of ions in the ${ }^{1} \mathrm{E}_{1}^{\prime}$ and ${ }^{3} \mathrm{E}_{1}^{\prime}$ states of configuration $\left(\left(a_{2}^{\prime \prime}\right)^{1}\left(e_{1}^{\prime \prime}\right)^{3}\left(e_{2}^{\prime \prime}\right)^{0}\right)$. According to Eq. (7) the ${ }^{1} \mathrm{E}_{1}^{\prime}$ state of the latter configuration and the ${ }^{1} \mathrm{E}_{2}^{\prime}$ state are vibronically coupled by modes of symmetry $\mathrm{e}_{1}^{\prime} \otimes \mathrm{e}_{2}^{\prime}=\mathrm{e}_{1}^{\prime}+\mathrm{e}_{2}^{\prime}$. Consequently, the $\mathrm{e}_{2}^{\prime}$ modes that mediate the strong PJT effect also induce a Herzberg-Teller intensity borrowing that enables the observation of the transitions to the ${ }^{1} \mathrm{E}_{2}^{\prime}$ state. This mechanism primarily lends intensity to the vibronic levels of symmetry $\mathrm{e}_{1}^{\prime}$ or $\mathrm{e}_{2}^{\prime}$ with $j>0$ but not to the $j=0$ level which is of $\mathrm{a}_{1}^{\prime}$ vibronic symmetry [34].

Our investigations of the cyclopentadienyl cation by high-resolution photoelectron spectroscopy provide the following picture of the structure and dynamics of this prototypical molecule. The ground state is of ${ }^{3} \mathrm{~A}_{2}^{\prime}$ symmetry and has a minimum energy geometry belonging to the $\mathrm{D}_{5 \mathrm{~h}}$ point group. This result contrasts with the naive expectation that an antiaromatic molecule should have C-C bonds of unequal lengths in its ground electronic state. The first excited electronic state is of symmetry ${ }^{1} \mathrm{E}_{2}^{\prime}$. It is subject to a strong PJT interaction with the next higher singlet state of symmetry ${ }^{1} \mathrm{~A}_{1}^{\prime}$ which stabilizes its lower component by $\sim 4000 \mathrm{~cm}^{-1}$. The potential energy surface of the lower component of the ${ }^{1} \mathrm{E}_{2}^{\prime}$ state is thus characterized by a deep trough (as shown schematically in Fig. 3) that gives rise to free pseudorotation. In the cyclopentadienyl cation, the PJT effect thus causes a high fluxionality of the molecule by allowing a complete delocalization of the nuclear wave function. 
This insight allows us to precisely describe the implications of antiaromaticity on a molecule. The triplet electronic state resulting from the lowest lying $\left(\mathrm{e}^{2}\right)$ electronic configuration does not distort because it is nondegenerate and no other triplet state results from that configuration, and all $\mathrm{C}-\mathrm{C}$ bond lengths are equal. The three singlet states (one $\mathrm{A}_{1}^{\prime}$ state and the two components of the $\mathrm{E}_{2}^{\prime}$ state) may distort to geometries with unequal $\mathrm{C}-\mathrm{C}$ bond lengths, but only through PJT interactions (see Fig. 5). The corresponding stabilization may lower one singlet state below the triplet state, as is the case in cyclobutadiene $[51,52]$. In the cyclopentadienyl cation, the stabilization energy is not large enough to lower the singlet state below the triplet state which results in an undistorted triplet ground state. The chemical intuition for an antiaromatic molecule is thus only applicable to the lowest singlet state of these molecules. In this state, $\mathrm{C}_{5} \mathrm{H}_{5}^{+}$distorts to form a dienylic and an allylic structure (see Fig. 5 b) which results in electronic localization and nuclear delocalization. The PJT effect can thus be interpreted as the way the molecule takes to avoid the unfavorable antiaromatic electronic structure.

\subsection{Methane cation}

The single-photon PFI-ZEKE photoelectron spectrum of methane is shown in Fig. 11 (top trace). Inspite of its simple appearance the rotational structure of this spectrum has long remained unassigned after its first observation in 1999 [53]. An experimental assignment of the nuclear spin symmetries using infrared-VUV double-resonance methods has eventually permitted the assignment of the spectrum and enabled the characterization of the dynamics induced by the JT effect $[29,41]$. The experimental technique has been described in Section 3 and consisted of two complementary methods. The first relied on fixing the VUV laser wave number at the position of a selected line of the PFI-ZEKE photoelectron spectrum and using an IR laser to saturate a rovibrational transition in the ground electronic state of the neutral molecule. A decrease of the PFI-ZEKE photoelectron signal indicated that the two transitions shared, as common initial state, the lower state of the IR transition. This method allowed the identification (presented as symmetry labels above the lines of the top spectrum in Fig. 11) of the rotational level of the vibronic ground state of methane from which the PFI-ZEKE transition originated. The second method consisted in exciting a specific rotational level of a vibrationally excited level of the neutral molecule and recording the PFI-ZEKE photoelectron spectrum from that selected level. ${ }^{12} \mathrm{CH}_{4}$ and ${ }^{12} \mathrm{CH}_{4}^{+}$possess three nuclear spin isomers with the nuclear spin symmetries in $\mathrm{T}_{\mathrm{d}}(\mathrm{M}) \mathrm{A}_{1}$ (meta, $I=2$ ), $\mathrm{E}$ (para, $I=0$ ) and $\mathrm{F}_{2}$ (ortho, $I=1$ ) $[54,55]$. Meta methane exists in levels of rovibronic symmetry $A_{1}$ or $A_{2}$, para methane in levels of 
symmetry $\mathrm{E}$ and ortho methane in levels of symmetry $\mathrm{F}_{1}$ or $\mathrm{F}_{2}$. Under the jet-cooled conditions of our experiments, only the three lowest rotational levels of the neutral vibronic ground state are populated, corresponding to the rotational quantum numbers and nuclear spin symmetries $J=0\left(\mathrm{~A}_{1}\right), J=1\left(\mathrm{~F}_{2}\right)$ and $J=2(\mathrm{E})$. Because electric dipole transitions only connect levels of the same nuclear spin symmetry, the identification of the initial state of the PFI-ZEKE photoelectron transition permits the assignment of the nuclear spin symmetry in the cation, i.e. the rovibronic symmetry without the index $\left(\mathrm{A}_{1}\right.$ and $\mathrm{A}_{2}$ are not distinguished, nor $\mathrm{F}_{1}$ and $\left.\mathrm{F}_{2}\right)$. In the case of a resonant two-photon excitation, the first transition can only populate levels of the same nuclear spin symmetry as the initial level and the same principle applies. The spectra obtained following two-photon resonant excitation are displayed in the four lower traces of Fig. 11. The knowledge of the initial state allows one to construct a map of the energy levels on an absolute energy scale, sorted by nuclear spin symmetry and to compare it with calculations. The result is displayed in Fig. 12 where the left-hand side represents the experimentally determined level positions with respect to the neutral rovibronic ground state, and the right-hand side the results of a calculation using an effective rotation-tunneling Hamiltonian [29,41]. There is a one-to-one correspondence between the calculated and experimental values of the levels of A and E symmetry up to $N^{+}=3$. The calculations predict more F levels than observed experimentally, but the grouping of calculated F levels reflects the experimental structure. It is likely that not all levels could be observed as a result of the resolution and sensitivity limits of the experiment. The adjustable parameters in the model Hamiltonian of Refs. [29,41] were three rotational constants $\left(A=6.40(13) \mathrm{cm}^{-1}, B=5.55(9) \mathrm{cm}^{-1}, C=4.03(6) \mathrm{cm}^{-1}\right)$ that essentially correspond to the rotational constants of the distorted $\mathrm{C}_{2 \mathrm{v}}$ structure of the potential minima, the tunneling splitting $\left(\delta=4|\sigma|=16.4(40) \mathrm{cm}^{-1}\right)$ and the ionization energy $E_{I} /(h c)=101752(15)$ $\mathrm{cm}^{-1}$. The comparison between the calculations and the experiment suggests that the effective rotation-tunneling Hamiltonian captures the essential aspects of the dynamics of the molecule and correctly incorporates the Jahn-Teller effect. The structure and dynamics of the ground state of $\mathrm{CH}_{4}^{+}$are characterized by a large-amplitude tunneling motion connecting equivalent minima of $\mathrm{C}_{2 \mathrm{v}}$ geometry. The $\mathrm{N}^{+}=0$ ground state of $\mathrm{CH}_{4}^{+}$is a tunneling doublet of triply degenerate levels and the lowest level has nuclear spin symmetry $\mathrm{F}_{2}$ (also $\mathrm{F}_{2}$ rovibronic symmetry according to theoretical considerations).

The results of our investigations of the methane cation by high-resolution photoelectron spectroscopy can thus be summarized in terms of a dynamic structure. The JT effect causes a distortion of the molecule away from the tetrahedral symmetry towards a distorted structure of 
$\mathrm{C}_{2 \mathrm{v}}$ symmetry. Rather than a single minimum, there are two enantiomeric sets of six equivalent minima and the molecule may be described as having a highly fluxional structure resulting from the tunneling between the structures of each set on the picosecond time scale. The sequence of tunneling levels, $F_{2}$ below $F_{1}$, is characteristic of the JT effect in a threefold degenerate electronic state, and results from a geometric phase effect [29]. The level structure for tunneling between six equivalent minima on a non-degenerate electronic surface (as in the case of a PJT effect with an electronically non-degenerate lower state) would be $A_{1}, F_{2}$, E.

Without the $\mathrm{JT}$ effect, $\mathrm{CH}_{4}^{+}$would have a rigid tetrahedral structure and the electron hole in the valence shell would be delocalized over three equivalent molecular orbitals of symmetry $\mathrm{f}_{2}$ (aligned along the three $\mathrm{C}_{2}$ axes of the molecule). In the presence of the JT effect, the electron hole is localized in a single molecular orbital (see Fig. 6) while the nuclei are delocalized over 12 equivalent potential energy minima of $\mathrm{C}_{2 \mathrm{v}}$ symmetry. The methane cation shares several common properties with the cyclopentadienyl cation. The JT effect induces a high fluxionality of the molecular structure and causes simultaneously an electronic localization and a nuclear delocalization. In both cases, the JT (or PJT) distortions can be rationalized by chemical intuition: in the case of the ${ }^{1} \mathrm{E}_{2}^{\prime}$ state of the cyclopentadienyl cation the distortion enables the molecule to avoid the unfavorable delocalized antiaromatic electronic structure by forming dienylic and allylic structures. In the case of $\mathrm{CH}_{4}^{+}$, the distortion towards a $\mathrm{CH}_{2}^{+}-\mathrm{H}_{2}$ complex (see Fig. 6) can be understood by consideration of the low ionization energy of $\mathrm{CH}_{2}$ [56].

\section{Isomerism and Chirality}

\subsection{Bond-stretch isomerism in $\mathrm{CH}_{3} \mathrm{D}^{+}$and $\mathrm{CH}_{2} \mathrm{D}_{2}^{+}$}

Partial isotopic substitution reduces the symmetry of the vibrational Hamiltonian without affecting the electronic degrees of freedom. It provides additional insights into vibronic coupling problems because the permutational symmetry is reduced and vibrational zero-point energy effects render otherwise equivalent minima distinguishable. Partial isotopic substitution of JT systems has been a very useful tool in determining the distorted equilibrium structure of molecules undergoing an $\mathrm{E} \otimes \mathrm{e}$ Jahn-Teller effect like the cyclooctatetraene anion [57], the benzene anion [58], benzene [59] and the cyclopentadienyl radical [60].

Partial deuteration of $\mathrm{CH}_{4}^{+}$removes the equivalence of the six minima represented in Fig. 8 because of different zero-point energies of the corresponding structures. If the hydrogen atom labeled " 4" in Fig. 8 is replaced by a deuterium atom, the structures 1, 2 and 3 remain equivalent, 
just as minima 4, 5 and 6 but the two sets are distinguishable. In the treatment that led to Eq. (21), one must therefore consider the following tunneling matrix elements:

$$
\begin{aligned}
\left\langle\chi_{i}|\hat{H}| \chi_{i}\right\rangle & =0 \text { for } i \in\{1,2,3\} \\
\left\langle\chi_{i}|\hat{H}| \chi_{i}\right\rangle & =\Delta \text { for } i \in\{4,5,6\} \\
1 / 2\left\langle\chi_{i}|\hat{H}| \chi_{j}\right\rangle & =\sigma \text { for } i \neq j,\{i, j\} \in\{1,2,3\} \\
1 / 2\left\langle\chi_{i}|\hat{H}| \chi_{j}\right\rangle & =\sigma^{\prime} \text { for } i \neq j,\{i, j\} \in\{4,5,6\} \\
1 / 2\left\langle\chi_{i}|\hat{H}| \chi_{j}\right\rangle & =\sigma^{\prime \prime} \text { for } i \neq j, i \in\{1,2,3\}, j \in\{4,5,6\} \text { or vice versa, }
\end{aligned}
$$

where $i$ and $j$ refer to the structures drawn in Fig. 8. $\sigma, \sigma^{\prime}$ and $\sigma^{\prime \prime}$ are all negative and $\Delta$ corresponds to the zero-point energy difference between the structures of both sets. The tunneling matrix for $\mathrm{CH}_{3} \mathrm{D}^{+}$thus takes the form ${ }^{b}$

and has the eigenvalues

$$
\mathbf{H}_{\mathrm{vib}}=\left(\begin{array}{cccccc}
0 & \sigma & -\sigma & \sigma^{\prime \prime} & -\sigma^{\prime \prime} & 0 \\
\sigma & 0 & \sigma & 0 & -\sigma^{\prime \prime} & \sigma^{\prime \prime} \\
-\sigma & \sigma & 0 & -\sigma^{\prime \prime} & 0 & \sigma^{\prime \prime} \\
\sigma^{\prime \prime} & 0 & -\sigma^{\prime \prime} & \Delta & \sigma^{\prime} & \sigma^{\prime} \\
-\sigma^{\prime \prime} & -\sigma^{\prime \prime} & 0 & \sigma^{\prime} & \Delta & \sigma^{\prime} \\
0 & \sigma^{\prime \prime} & \sigma^{\prime \prime} & \sigma^{\prime} & \sigma^{\prime} & \Delta
\end{array}\right)
$$

$$
\begin{aligned}
E_{\mathrm{E}} & =\frac{1}{2}\left(\sigma-\sigma^{\prime}+\Delta-S\right) \\
E_{\mathrm{A}_{2}} & =-2 \sigma \\
E_{\mathrm{A}_{1}} & =2 \sigma^{\prime}+\Delta \\
E_{\mathrm{E}} & =\frac{1}{2}\left(\sigma-\sigma^{\prime}+\Delta+S\right) .
\end{aligned}
$$

In Eqs. (25a)-(25d), the symmetry labels given as subscripts correspond to the irreducible representations of the corresponding eigenvectors in $\mathrm{C}_{3 \mathrm{v}}(\mathrm{M})$ and ${ }^{c}$

$$
S=\sqrt{\left(\sigma+\sigma^{\prime}\right)^{2}+12 \sigma^{\prime \prime 2}-2\left(\sigma+\sigma^{\prime}\right) \Delta+\Delta^{2}} .
$$

In the limit $\left(|\sigma|,\left|\sigma^{\prime}\right|,\left|\sigma^{\prime \prime}\right|\right) \ll \Delta$ one obtains

$$
S \approx \Delta-\left(\sigma+\sigma^{\prime}\right)+\frac{\left(\sigma+\sigma^{\prime}\right)^{2}+12 \sigma^{\prime \prime 2}}{2 \Delta} \approx \Delta-\left(\sigma+\sigma^{\prime}\right),
$$

\footnotetext{
${ }^{b}$ Eq. (4) of Ref. [61] represents the tunneling matrix corresponding to Fig. 1 of that article, but with structures 4 and 5 reversed.

${ }^{c}$ Note that Eqs. (6) and (7) in Ref. [61] have been mistyped.
} 
and the eigenvalues converge to

$$
\begin{aligned}
E_{\mathrm{E}} & =\sigma \\
E_{\mathrm{A}_{2}} & =-2 \sigma \\
E_{\mathrm{A}_{1}} & =2 \sigma^{\prime}+\Delta \\
E_{\mathrm{E}} & =-\sigma^{\prime}+\Delta,
\end{aligned}
$$

corresponding to two tunneling pairs $\left(\left(\mathrm{E}, \mathrm{A}_{2}\right)\right.$ and $\left(\mathrm{A}_{1}, \mathrm{E}\right)$, respectively) with tunneling splittings $\delta=3|\sigma|$ and $\delta^{\prime}=3\left|\sigma^{\prime}\right|$, respectively. The tunneling states are represented schematically in Fig. 13. If $|\Delta|$ is large compared to the tunneling matrix elements as is the case in $\mathrm{CH}_{3} \mathrm{D}^{+}$, the interconversion of one of the equilibrium structures 1,2 and 3 to one of the structures 4,5 and 6 is suppressed and two isomers exist. Minima 1, 2 and 3 have a short C-D bond and can be designated by the label $\mathrm{CH}_{3} \mathrm{D}_{s}^{+}$whereas minima 4,5 and 6 have a long C-D bond and are designated by $\mathrm{CH}_{3} \mathrm{D}_{\ell}^{+}$.

The vibronic symmetries can also be predicted by group theory as outlined in Section 2 . The ordering of the orbitals and the shape of the singly occupied molecular orbital of $\mathrm{CH}_{4}^{+}$ is not significantly affected by deuteration. Classification of the electronic symmetry of this orbital in the $\mathrm{C}_{\mathrm{s}}$ point group appropriate to $\mathrm{CH}_{3} \mathrm{D}^{+}$at the minimum energy geometry gives $\mathrm{A}^{\prime \prime}$ for $\mathrm{CH}_{3} \mathrm{D}_{s}^{+}$where the plane of symmetry contains the two short bonds and $\mathrm{A}^{\prime}$ for $\mathrm{CH}_{3} \mathrm{D}_{\ell}^{+}$ where the plane of symmetry contains the two long bonds. By correlating these symmetries to the molecular symmetry group corresponding to feasible permutations of the three protons $\left(\mathrm{C}_{3 \mathrm{v}}(\mathrm{M})\right)$ the tunneling splittings $\mathrm{A}_{2} \oplus \mathrm{E}$ for $\mathrm{CH}_{3} \mathrm{D}_{s}^{+}$and $\mathrm{A}_{1} \oplus \mathrm{E}$ for $\mathrm{CH}_{3} \mathrm{D}_{\ell}^{+}$are predicted in accordance with the tunneling calculations described above.

The PFI-ZEKE photoelectron spectrum of $\mathrm{CH}_{3} \mathrm{D}$ is displayed in Fig. 14. The bands labeled $\mathrm{A}$ and $\mathrm{B}$ have been assigned to the two isomers $\mathrm{CH}_{3} \mathrm{D}_{s}^{+}$and $\mathrm{CH}_{3} \mathrm{D}_{\ell}^{+}$, respectively, on the basis of their tunnel-rotational structures [61]. Band A consists of transitions to a tunneling doublet of vibronic levels $\mathrm{E}$ and $\mathrm{A}_{2}$ whereas band $\mathrm{B}$ consists of transitions to a tunneling doublet $\mathrm{A}_{1}$ and E. The experimentally determined zero-point energy difference amounts to $121(1) \mathrm{cm}^{-1}$ which agrees with the value of $114 \mathrm{~cm}^{-1}$ calculated at the $\operatorname{CCSD}(\mathrm{T}) / \mathrm{cc}-\mathrm{pVTZ}$ level of ab initio theory. The tunneling splittings (equal to $\left|3 \sigma^{(\prime)}\right|$ ) are much smaller and amount to 9.6 and $6.6 \mathrm{~cm}^{-1}$, respectively, as determined from the experimental spectrum. In each of these isomers, the hydrogen atoms exchange on a picosecond time scale and are therefore equivalent on the time scale of our experiments. Hence, $\mathrm{CH}_{3} \mathrm{D}^{+}$indeed appears to possess two distinct isomers that must have different chemical properties. 
The symmetry is lowered even further if two hydrogen atoms are replaced by deuterium atoms. In the treatment that led to Eq. (21), one must therefore consider the following tunneling matrix elements:

$$
\begin{aligned}
\left\langle\chi_{i}|\hat{H}| \chi_{i}\right\rangle & =0 \text { for } i=1 \\
\left\langle\chi_{i}|\hat{H}| \chi_{i}\right\rangle & =\Delta \text { for } i \in\{2,3,4,5\} \\
\left\langle\chi_{i}|\hat{H}| \chi_{i}\right\rangle & =\Delta^{\prime} \text { for } i=6 \\
1 / 2\left\langle\chi_{i}|\hat{H}| \chi_{j}\right\rangle & =\sigma \text { for } i \neq j,\{i, j\} \in\{2,3,4,5\} \\
1 / 2\left\langle\chi_{i}|\hat{H}| \chi_{j}\right\rangle & =\sigma^{\prime} \text { for } i \neq j, i=1, j \in\{2,3,4,5\} \text { or vice versa, } \\
1 / 2\left\langle\chi_{i}|\hat{H}| \chi_{j}\right\rangle & =\sigma^{\prime \prime} \text { for } i \neq j, i=6, j \in\{2,3,4,5\} \text { or vice versa, }
\end{aligned}
$$

where $i$ and $j$ refer to the structures drawn in Fig. 8. $\sigma, \sigma^{\prime}$ and $\sigma^{\prime \prime}$ are all negative. $\Delta$ and $\Delta^{\prime}$ correspond to the zero-point energy difference between the structures 1 and 2-5 and 1 and 6 , respectively. The tunneling matrix for $\mathrm{CH}_{2} \mathrm{D}_{2}^{+}$thus takes the form

$$
\mathbf{H}_{\mathrm{tun}}=\left(\begin{array}{cccccc}
0 & \sigma^{\prime} & -\sigma^{\prime} & \sigma^{\prime} & -\sigma^{\prime} & 0 \\
\sigma^{\prime} & \Delta & \sigma & 0 & -\sigma & \sigma^{\prime \prime} \\
-\sigma^{\prime} & \sigma & \Delta & -\sigma & 0 & \sigma^{\prime \prime} \\
\sigma^{\prime} & 0 & -\sigma & \Delta & \sigma & \sigma^{\prime \prime} \\
-\sigma^{\prime} & -\sigma & 0 & \sigma & \Delta & \sigma^{\prime \prime} \\
0 & \sigma^{\prime \prime} & \sigma^{\prime \prime} & \sigma^{\prime \prime} & \sigma^{\prime \prime} & \Delta^{\prime}
\end{array}\right),
$$

and has the eigenvalues (and approximate values in the case $\left.\left(\left|\sigma^{\prime}\right|,\left|\sigma^{\prime \prime}\right|\right) \ll\left(\Delta, \Delta^{\prime}\right)\right)$

$$
\begin{aligned}
& E_{\mathrm{B}_{2}}=\frac{1}{2}\left(\Delta-S_{1}\right) \approx-\frac{4 \sigma^{\prime 2}}{\Delta} \\
& E_{\mathrm{A}_{1}}=2 \sigma+\Delta \\
& E_{\mathrm{B}_{1}}=\frac{1}{2}\left(\Delta+\Delta^{\prime}-S_{2}\right) \approx \Delta-\frac{4 \sigma^{\prime 2}}{\Delta^{\prime}-\Delta} \\
& E_{\mathrm{B}_{2}}=\frac{1}{2}\left(\Delta+S_{1}\right) \approx \Delta+\frac{4 \sigma^{\prime 2}}{\Delta} \\
& E_{\mathrm{A}_{2}}=-2 \sigma+\Delta \\
& E_{\mathrm{B}_{1}}=\frac{1}{2}\left(\Delta+\Delta^{\prime}+S_{2}\right) \approx \Delta^{\prime}+\frac{4 \sigma^{\prime \prime 2}}{\Delta^{\prime}-\Delta},
\end{aligned}
$$

where

$$
\begin{aligned}
& S_{1}=\sqrt{\left(\Delta^{2}+16 \sigma^{\prime 2}\right)} \\
& S_{2}=\sqrt{\left(\Delta^{2}-2 \Delta \Delta^{\prime}+\Delta^{\prime 2}+16 \sigma^{\prime \prime 2}\right)} .
\end{aligned}
$$


In the limit $\left(\left|\sigma^{\prime}\right|,\left|\sigma^{\prime \prime}\right|\right) \ll\left(\Delta, \Delta^{\prime}\right)$, the eigenvalues become

$$
\begin{aligned}
& E_{\mathrm{B}_{2}}=0 \\
& E_{\mathrm{A}_{1}}=2 \sigma+\Delta \\
& E_{\mathrm{B}_{1}}=\Delta \\
& E_{\mathrm{B}_{2}}=\Delta \\
& E_{\mathrm{A}_{2}}=-2 \sigma+\Delta \\
& E_{\mathrm{B}_{1}}=\Delta^{\prime},
\end{aligned}
$$

and the corresponding eigenvectors are

$$
\mathbf{U}=\frac{1}{2}\left(\begin{array}{cccccc}
2 & 0 & 0 & 0 & 0 & 0 \\
0 & -1 & 1 & -1 & 1 & 0 \\
0 & -1 & 1 & 1 & -1 & 0 \\
0 & 1 & 1 & -1 & -1 & 0 \\
0 & 1 & 1 & 1 & 1 & 0 \\
0 & 0 & 0 & 0 & 0 & 2
\end{array}\right),
$$

where the columns represent the eigenvectors in the same order as the eigenvalues given in Eq. (33). The first and the last eigenvalues correspond to eigenvectors that are localized in minima 1 and 6, belonging to the isomers $\mathrm{CH}_{\ell} \mathrm{H}_{\ell} \mathrm{D}_{s} \mathrm{D}_{s}^{+}$and $\mathrm{CH}_{s} \mathrm{H}_{s} \mathrm{D}_{\ell} \mathrm{D}_{\ell}^{+}$, respectively. The four intermediate eigenvalues correspond to the isomer $\mathrm{CH}_{\ell} \mathrm{H}_{s} \mathrm{D}_{\ell} \mathrm{D}_{s}^{+}$as shown schematically in Fig. 15.

Figure 16 shows the PFI-ZEKE photoelectron spectrum of $\mathrm{CH}_{2} \mathrm{D}_{2}$ in the region of the adiabatic ionization threshold. Three bands labeled A, B and C can be distinguished that possess a very different (tunnel-)rotational structure. The separation between the centers of the bands A and B amounts to approximately $130 \mathrm{~cm}^{-1}$ and the separation between the bands $\mathrm{A}$ and $\mathrm{C}$ is $240 \mathrm{~cm}^{-1}$. The calculated difference in zero-point energies between the isomers $\mathrm{CH}_{\ell} \mathrm{H}_{\ell} \mathrm{D}_{s} \mathrm{D}_{s}^{+}$and $\mathrm{CH}_{\ell} \mathrm{H}_{s} \mathrm{D}_{\ell} \mathrm{D}_{s}^{+}$is $115 \mathrm{~cm}^{-1}$ and that between $\mathrm{CH}_{\ell} \mathrm{H}_{\ell} \mathrm{D}_{s} \mathrm{D}_{s}^{+}$and $\mathrm{CH}_{\ell} \mathrm{H}_{s} \mathrm{D}_{\ell} \mathrm{D}_{s}^{+}$is $220 \mathrm{~cm}^{-1}$ at the UMP2/cc-pVDZ level of ab initio theory. Consequently, the attribution of bands $\mathrm{A}, \mathrm{B}$ and $\mathrm{C}$ to the three isomers appears justified.

The isomers $\mathrm{CH}_{\ell} \mathrm{H}_{\ell} \mathrm{D}_{s} \mathrm{D}_{s}^{+}$and $\mathrm{CH}_{s} \mathrm{H}_{s} \mathrm{D}_{\ell} \mathrm{D}_{\ell}^{+}$possess $\mathrm{C}_{2 \mathrm{v}}$ symmetry and their ground electronic states have ${ }^{2} \mathrm{~B}_{2}$ and ${ }^{2} \mathrm{~B}_{1}$ symmetry, respectively. Because these isomers are "rigid" on the time scale of our measurements [62], their ground vibronic state must also have $\mathrm{B}_{2}\left(\mathrm{~B}_{1}\right)$ symmetry, in agreement with results of the tunneling calculations. The structure of isomer 
$\mathrm{CH}_{\ell} \mathrm{H}_{s} \mathrm{D}_{\ell} \mathrm{D}_{s}^{+}$has no symmetry elements and therefore belongs to the $\mathrm{C}_{1}$ point group. Since the tunneling motion connecting the four equivalent minima (2, 3, 4 and 5 in Fig. 8) is feasible, the CNPI of this isomer is $\mathrm{C}_{2 \mathrm{v}}(\mathrm{M})$. $\mathrm{C}_{1}$ possesses a single irreducible representation that correlates with $\mathrm{A}_{1} \oplus \mathrm{A}_{2} \oplus \mathrm{B}_{1} \oplus \mathrm{B}_{2}$ in $\mathrm{C}_{2 \mathrm{v}}(\mathrm{M})$ and the vibronic symmetries resulting from tunneling correspond to the four irreducible representations of $\mathrm{C}_{2 \mathrm{v}}(\mathrm{M})$ as obtained above.

\subsection{The Jahn-Teller effect and chirality in $\mathrm{C}_{2} \mathrm{H}_{4}^{+}$and $\mathrm{CH}_{2} \mathrm{D}_{2}^{+}$}

The parity violating nature of the electro-weak interaction is responsible for a small energy difference between two enantiomeric structures $[38,39,63,64]$. The influence of this effect on the dynamics of a chiral molecule depends on the relative magnitude of the parity violating effect and the size of the tunneling splitting associated with stereomutation.

In many chiral molecules, like methane derivatives with four different substituents or heavier analogues of hydrogen peroxide, tunneling splittings can be very small and two cases must be distinguished $[39,63]$ : i) If the parity-violating energy difference between the two enantiomers is much smaller that the splitting resulting from tunneling, the latter dominates the dynamics of the molecule in its ground state. The vibronic ground state of the molecule is delocalized over both minima of the potential energy surface. ii) If the tunneling splitting is much smaller than the parity-violating energy difference, the latter causes a localization of the vibronic ground state in the potential energy minimum corresponding to the more stable enantiomer. The next higher vibronic state is localized in the minimum corresponding to the other enantiomer. In this case the parity-violating interaction effectively breaks the symmetry of the molecule. The energy difference between the two enantiomeric structures induced by the electro-weak interactions effectively suppresses tunneling just like the difference in zero-point energies suppresses tunneling between minima corresponding to different isomers in $\mathrm{CH}_{3} \mathrm{D}^{+}$.

Distortions of the molecular geometry by the JT effect can induce chirality and this subsection discusses the two cases of $\mathrm{C}_{2} \mathrm{H}_{4}^{+}$and $\mathrm{CH}_{2} \mathrm{D}_{2}^{+}$. The ethylene cation has a non-planar equilibrium structure $[65,66]$. The lowest potential energy surfaces are represented schematically in Fig. 17. Using the planar $\mathrm{D}_{2 \mathrm{~h}}$ geometry as reference, the nonplanarity is a result of the vibronic coupling between the $\tilde{\mathrm{X}}^{2} \mathrm{~B}_{3 \mathrm{u}}$ and $\tilde{\mathrm{A}}{ }^{2} \mathrm{~B}_{3 \mathrm{~g}}$ mediated by the torsional mode of irreducible representation $A_{u}$. Alternatively, the distortion can also be considered to result from a JT effect that splits the ground $\tilde{\mathrm{X}}^{2} \mathrm{E}$ state of $\mathrm{D}_{2 \mathrm{~d}}$ geometry into two components of symmetry ${ }^{2} \mathrm{~B}_{3}$ and ${ }^{2} \mathrm{~B}_{2}$ in $\mathrm{D}_{2}$ geometry. The ${ }^{2} \mathrm{~B}_{3}$ component becomes the $\tilde{\mathrm{X}}^{2} \mathrm{~B}_{3 \mathrm{u}}$ state at the planar geometry, whereas the ${ }^{2} \mathrm{~B}_{2}$ component becomes the $\tilde{\mathrm{C}}^{2} \mathrm{~B}_{2 \mathrm{u}}$ state (not shown). 
Assuming that the barriers at both the $\mathrm{D}_{2 \mathrm{~h}}$ and the $\mathrm{D}_{2 \mathrm{~d}}$ geometry are impenetrable, the appropriate symmetry group for $\mathrm{C}_{2} \mathrm{H}_{4}^{+}$is $\mathrm{D}_{2}$. The electronic ground state is of ${ }^{2} \mathrm{~B}_{3}$ geometry. Assuming that tunneling through the barrier at planarity $\left(\mathrm{D}_{2 \mathrm{~h}}\right.$ geometry $)$ is feasible changes the appropriate molecular symmetry group to $D_{2 h}(M)$, and the ground vibronic state splits into the tunneling doublet $\mathrm{B}_{3 \mathrm{u}} \oplus \mathrm{B}_{3 \mathrm{~g}}$ which has indeed been observed experimentally [66]. If, on the contrary, tunneling were only possible through the barrier at $\mathrm{D}_{2 \mathrm{~d}}$ geometry, the appropriate molecular symmetry group would be $\mathrm{D}_{2 \mathrm{~d}}(\mathrm{M})$, and the vibronic ground state would have $\mathrm{E}$ symmetry. Therefore, even if the barrier at $\mathrm{D}_{2 \mathrm{~d}}$ geometry were very low, there would not be any signature of tunneling. This situation is encountered in the allene cation [67].

Since tunneling through the barrier at the $\mathrm{D}_{2 \mathrm{~d}}$ geometry can be ignored, the dynamics of $\mathrm{C}_{2} \mathrm{H}_{4}^{+}$can be qualitatively described by the two-minimum potential shown in Fig. 17 . The minimum energy structure has $\mathrm{D}_{2}$ geometry and is chiral in the same way as $\mathrm{H}_{2} \mathrm{O}_{2}, \mathrm{H}_{2} \mathrm{~S}_{2}, \mathrm{Cl}_{2} \mathrm{~S}_{2}$, etc. are chiral [68-70]. The structures corresponding to two adjacent minima are enantiomers of each other. The lowering of symmetry resulting from vibronic coupling thus induces chirality in a system that would otherwise be achiral. In the ethylene cation, the splitting associated with the tunneling motion through the $\mathrm{D}_{2 \mathrm{~h}}$ barrier amounts to $83.7(5) \mathrm{cm}^{-1}$ which corresponds to a stereomutation time of 199 fs. This motion clearly dominates the dynamics of the ground state of $\mathrm{C}_{2} \mathrm{H}_{4}^{+}$and the separation of enantiomers is not possible. $\mathrm{C}_{2} \mathrm{H}_{4}^{+}$clearly belongs to the first category of chiral molecules mentioned above. Indeed, the energy difference between the two enantiomeric structures of $\mathrm{C}_{2} \mathrm{H}_{4}^{+}$caused by the electro-weak interaction is expected to be on the order of $10^{-13} \mathrm{~cm}^{-1}$ (which is the order of magnitude that was calculated for allene [71]). Substitution of the $\mathrm{H}$ atoms in $\mathrm{C}_{2} \mathrm{H}_{4}^{+}$by heavier atoms may, however, provide further examples of molecules in the second category mentioned above.

The substituted methane derivatives represent an important class of chiral molecules [38]. Methane itself is not chiral in its ground vibronic state but its stereomutation, i.e., the process that converts a structure with four numbered hydrogen atoms into its enantiomer, is important in understanding the dynamics of this class of chiral molecules. In the ground state of $\mathrm{CH}_{4}$, the tunneling splitting resulting from this motion is expected to be very small because of the very high barrier $\left(\geq 35000 \mathrm{~cm}^{-1}\right.$ ) for stereomutation [72]. In $\mathrm{CH}_{4}^{+}$, the barrier for stereomutation is much lower [35]. We have calculated the lowest potential energy surface of $\mathrm{CH}_{4}^{+}$at the UMP2/cc-pVDZ level of $a b$ initio theory along the reaction path connecting a $\mathrm{C}_{2 \mathrm{v}}$ minimum energy structure to its enantiomeric structure using the algorithm implemented in Gaussian03 [73]. The maximum of the reaction path corresponds to a structure of $\mathrm{C}_{\mathrm{s}}$ geometry that is very 
similar to the one described in Ref. [35]. The purely electronic barrier height is found to be $5100 \mathrm{~cm}^{-1}$ (4700 $\mathrm{cm}^{-1}$ after harmonic zero-point correction). The estimation of the tunneling splitting associated with stereomutation was performed using a WKB method [74] and the calculated reaction path and gave the value $10^{-7} \mathrm{~cm}^{-1}$ for this splitting.

What are the spectroscopic manifestation of stereomutation tunneling in $\mathrm{CH}_{4}^{+}$? To obtain the appropriate molecular symmetry group, $\mathrm{T}_{\mathrm{d}}(\mathrm{M})$ has to be extended to include operations that interconvert enantiomeric structures. The appropriate group contains all permutation-inversion operations of four particles and is designated $S_{4}^{*}[38]$ (Ref. [75] uses a different notation). The correlation of irreducible representations including spin-statistical weights for $\mathrm{CH}_{4}^{+}$and $\mathrm{CD}_{4}^{+}$ are given in Tables III and IV, respectively. The tables show that in $\mathrm{CH}_{4}^{+}$, only the levels of rovibronic symmetry $\mathrm{E}$ will be split by stereomutation tunneling, whereas in $\mathrm{CD}_{4}^{+}$, the $\mathrm{E}$, the $F_{1}$ and the $F_{2}$ levels will be split. The statistical weights of + and - parity sublevels is equal in the $\mathrm{E}$ levels but different in the $\mathrm{F}_{1}$ and $\mathrm{F}_{2}$ levels.

The classification of chirality discussed so far is sufficient to describe the ground state dynamics of chiral molecules characterized by tunneling between two minima corresponding to enantiomeric structures. The isomer $\mathrm{CH}_{\ell} \mathrm{H}_{s} \mathrm{D}_{\ell} \mathrm{D}_{s}^{+}$of $\mathrm{CH}_{2} \mathrm{D}_{2}^{+}$represents a special case of chirality, where eight potential energy minima are isoenergetic if electro-weak interactions are ignored.

As explained in Section 4 in the discussion of $\mathrm{CH}_{4}^{+}$, one can expect that the potential energy barriers separating the four structures shown in Fig. 18 are much lower than the barrier for stereomutation. Consequently, the discussion will be restricted to four minima. As discussed in Section 5.1, tunneling between these four minima leads to a splitting of the four-fold degenerate ground vibronic state into a $1+2+1$ pattern where the vibronic symmetries are $A_{1}, B_{1}$, $\mathrm{B}_{2}$ and $\mathrm{A}_{2}$. An inspection of the structures displayed in Fig. 18 reveals that directly adjacent structures are enantiomers of each other. Structures at opposite vertices of the diagram are identical except for the numbering of their substituents. The enantiomers can be labeled according to the nomenclature of axially chiral compounds (e.g., the asymmetrically substituted allene derivatives) because the substituent positions are pairwise equivalent in $\mathrm{CH}_{2} \mathrm{D}_{2}^{+}$. The structure corresponding to minimum 2 would thus be labeled $(S)-\mathrm{CH}_{\ell} \mathrm{H}_{s} \mathrm{D}_{\ell} \mathrm{D}_{s}^{+}$whereas minimum 5 corresponds to $(R)-\mathrm{CH}_{\ell} \mathrm{H}_{s} \mathrm{D}_{\ell} \mathrm{D}_{s}^{+}$. Clearly, the fast pseudorotational tunneling connecting neighboring minima interconverts enantiomeric structures. In the following, we shall investigate how the tunneling dynamics affects the chirality of $\mathrm{CH}_{\ell} \mathrm{H}_{s} \mathrm{D}_{\ell} \mathrm{D}_{s}^{+}$that is induced by the JT effect.

A closer inspection of the vibronic eigenfunctions associated with the $B_{1}$ and $B_{2}$ levels (see Eq. (34)) shows that they correspond to symmetric and antisymmetric combinations of 
structures 2,4 and 3, 5, respectively. Indeed, the symmetric linear combination of the $\mathrm{B}_{1}$ and $\mathrm{B}_{2}$ functions reads $\frac{1}{\sqrt{2}}(0,1,0,1)$ and the antisymmetric combination is $\frac{1}{\sqrt{2}}(1,0,1,0)$ (where the numbers in parentheses are the coefficients of the wave function in the basis $\left\{\phi_{2}, \phi_{3}, \phi_{4}, \phi_{5}\right\}$ of localized vibronic functions analogous to that defined in Eq. (20) for $\mathrm{CH}_{4}^{+}$). The former wavefunction corresponds to a pure $(R)$-enantiomer and the latter to a pure $(S)$-enantiomer. A coherent superposition with the appropriate relative phase of the $B_{2}$ and $B_{1}$ vibronic levels would thus correspond to a single enantiomer.

We first consider the case where tunneling between inequivalent minima is negligible $\left(\sigma^{\prime}=\right.$ $\sigma^{\prime \prime}=0$ in Eq. (30)). In this case, the $\mathrm{B}_{1}$ and $\mathrm{B}_{2}$ levels are exactly degenerate and, consequently, the associated wave function can be chosen arbitrarily. Each enantiomer of $\mathrm{CH}_{\ell} \mathrm{H}_{s} \mathrm{D}_{\ell} \mathrm{D}_{s}^{+}$would have a very long stereomutation time and could be isolated. However, the tunneling between inequivalent minima removes this accidental degeneracy and results in a splitting $E\left(\mathrm{~B}_{2}\right)$ $E\left(\mathrm{~B}_{1}\right) \approx 4\left(\sigma^{\prime 2} / \Delta+\sigma^{\prime \prime 2} /\left(\Delta^{\prime}-\Delta\right)\right)$. The tunneling elements are expected to be on the order of $1 \mathrm{~cm}^{-1}$ whereas the zero point energy differences are close to $100 \mathrm{~cm}^{-1}$. The splitting of the nearly degenerate $B_{2}$ and $B_{1}$ levels will therefore be on the order of $0.1 \mathrm{~cm}^{-1}$. An investigation, at high resolution, of the tunneling-rotational structure of band B in the spectrum shown in Fig. 16 corresponding to the $\mathrm{CH}_{\ell} \mathrm{H}_{s} \mathrm{D}_{\ell} \mathrm{D}_{s}^{+}$isomer would thus reveal interesting details about the unusual dynamics of chirality in this molecule.

\section{A related non-Jahn-Teller fluxional system: $\mathrm{CH}_{5}^{+}$}

The group theoretical methods used above to predict the vibronic symmetries of tunneling levels in vibronically coupled systems have a broader range of applicability. They can also be applied to rovibronic states, are useful in the construction of correlation diagrams [41,61] and may be used to treat non-Jahn-Teller fluxional systems. An important molecule that is closely related to the methane cation is protonated methane $\left(\mathrm{CH}_{5}^{+}\right)$, which is similar to $\mathrm{CH}_{4}^{+}$in that its hydrogen atoms exchange on a (sub)-picosecond timescale. However, its ground electronic state has a closed-shell electronic structure, and $\mathrm{CH}_{5}^{+}$is therefore not subject to a JT effect. $\mathrm{CH}_{5}^{+}$has been of considerable interest to chemists because it is the prototype of hypercoordinated carbocations [76] and plays an important role in the chemistry of the interstellar medium [77]. Its infrared spectrum has been observed in laboratory experiments [78-80] but has not been conclusively assigned to date, though theoretical progress has been made recently towards establishing its vibrational energy level structure [81]. 
The lowest potential energy surface of $\mathrm{CH}_{5}^{+}$is very flat and possesses several stationary points that lie close in energy (see, e.g., Refs. [82-87]) and are represented schematically in Fig. 19. Recent calculations agree on a global minimum energy structure of $\mathrm{C}_{\mathrm{s}}$ symmetry and first order saddle points of symmetries $\mathrm{C}_{\mathrm{s}}$ and $\mathrm{C}_{2 \mathrm{v}}$ (the three structures are labeled $\mathrm{C}_{\mathrm{s}}(\mathrm{I})$, $\mathrm{C}_{\mathrm{s}}(\mathrm{II})$ and $\mathrm{C}_{2 \mathrm{v}}$ in Fig. 19 and their relative electronic potential energies are indicated) but the ordering may change depending on the magnitude of the applied zero-point energy correction. A tunneling description is thus probably not fully adequate for the ground electronic state of $\mathrm{CH}_{5}^{+}$. However, the strategy outlined in the present article, especially the group theoretical symmetry correlations, the determination of nuclear spin symmetries and partial isotopic substitution are expected to provide additional help in the assignment of the complex spectra of $\mathrm{CH}_{5}^{+}$and its partially deuterated isotopomers.

We first assume that structure $\mathrm{C}_{\mathrm{s}}(\mathrm{I})$ represented in Fig. 19 is the global minimum energy structure after zero-point correction. This structure belongs to the $\mathrm{C}_{\mathrm{s}}$ point group and since the molecule has a closed-shell electronic structure, the irreducible representation of its rovibronic symmetry can only be $\mathrm{A}^{\prime}$. The CNPI group of $\mathrm{CH}_{5}^{+}$is $\mathrm{G}_{240}=S_{5} \otimes\left\{E, E^{*}\right\}$. By correlating the appropriate $\mathrm{C}_{\mathrm{s}}$ point group to $\mathrm{G}_{240}$, one finds that tunneling between all equivalent minima splits the ground state into ten sublevels with non-zero spin statistical weights and degeneracies 1,4 and 5, respectively: $\mathrm{A}_{2}^{-}(6) \oplus \mathrm{G}_{2}^{+}(4) \oplus 3 \mathrm{G}_{2}^{-}(4) \oplus 2 \mathrm{H}_{2}^{+}(2) \oplus 3 \mathrm{H}_{2}^{-}(2)$. The lowest-lying rotationally excited level $\left(1_{01}\right.$ in asymmetric top notation, rovibronic symmetry $\mathrm{A}^{\prime \prime}$ in the $\mathrm{C}_{\mathrm{s}}$ point group) splits into $\mathrm{A}_{2}^{+}(6) \oplus 3 \mathrm{G}_{2}^{+}(4) \oplus \mathrm{G}_{2}^{-}(4) \oplus 3 \mathrm{H}_{2}^{+}(2) \oplus 2 \mathrm{H}_{2}^{-}(2)$ (see also Ref. [88]). If, on the contrary, the structure of $\mathrm{C}_{2 \mathrm{v}}$ symmetry is the global minimum, the splitting of the ground state resulting from tunneling between the equivalent minima will be different. In the $\mathrm{C}_{2 \mathrm{v}}$ point group, the symmetry of the rovibronic ground state must be $A_{1}$ and the correlation to $G_{240}$ gives $\mathrm{A}_{2}^{-}(6) \oplus 2 \mathrm{G}_{2}^{-}(4) \oplus \mathrm{H}_{2}^{+}(2) \oplus 2 \mathrm{H}_{2}^{-}(2)$, which is qualitatively different from the splitting obtained in the case of a minimum of $\mathrm{C}_{\mathrm{s}}$ symmetry. The determination of nuclear spin symmetries as we have carried out for $\mathrm{CH}_{4}^{+}$and the identification of the rovibronic symmetries resulting from tunneling would therefore simplify the assignment of the high-resolution infrared spectra $[78,80]$.

As shown above, partial isotopic substitution suppresses tunneling between inequivalent minima in the methane cation which leads to the existence of isomers: two in the case of $\mathrm{CH}_{3} \mathrm{D}^{+}$ and $\mathrm{CD}_{3} \mathrm{H}^{+}$and three in the case of $\mathrm{CH}_{2} \mathrm{D}_{2}^{+}$. In the case of $\mathrm{CH}_{5}^{+}$, assuming the $\mathrm{C}_{\mathrm{s}}$ structure (I) to be the minimum energy geometry (see Fig. 19), it is possible to predict the number of isomers. This structure possesses only two equivalent hydrogen atoms which are below and above the plane of Fig. 19, respectively. $\mathrm{CH}_{4} \mathrm{D}^{+}$and $\mathrm{CHD}_{4}^{+}$could thus possess 4 isomers. If 
the only $\mathrm{H}$ (or $\mathrm{D}$ ) atom occupies one of the two equivalent positions, a chiral structure results. $\mathrm{CH}_{3} \mathrm{D}_{2}^{+}$and $\mathrm{CH}_{2} \mathrm{D}_{3}^{+}$could possess 7 isomers. If the two equivalent positions are occupied by two different isotopes the structure is chiral. There may therefore be three different chiral structures for both $\mathrm{CH}_{3} \mathrm{D}_{2}^{+}$and $\mathrm{CH}_{2} \mathrm{D}_{3}^{+}$(without counting the enantiomers).

Whether partial deuteration of $\mathrm{CH}_{5}^{+}$induces isomerism or not depends on the heights of the barriers and the differences in the vibrational zero-point corrections. The purely electronic barrier heights are known from accurate ab initio calculations [86] and are indicated in Fig. 19. They are low compared to the case of $\mathrm{CH}_{4}^{+}$and therefore, the vibrational zero-point energy corrections are expected to become dominant. The calculations reported in Refs. [89-91] indicate that deuteration partially suppresses tunneling but the effect may be too small to induce an isomerism of the kind discussed above for the methane cation. Indeed, the vibrational wave functions may well have amplitude in minima corresponding to several distinct isomeric structures.

Partial deuteration could nevertheless facilitate the assignment of the infrared spectrum. First, the distinct stretching frequencies of the C-H and C-D chromophores will reduce spectral congestion. Moreover, the inequivalence of the different minima induced by different zero-pointenergy corrections leads to a partial localization of the nuclear wave functions and therefore a higher rigidity of the molecule. In the case of the methane cation, the inspection of the PFI-ZEKE photoelectron spectra of $\mathrm{CH}_{2} \mathrm{D}_{2}^{+}$and $\mathrm{CH}_{3} \mathrm{D}^{+}$immediately reveals the existence of three and two isomers, respectively, which establishes the $\mathrm{C}_{2 \mathrm{v}}$ symmetry of the minimum energy structure.

\section{Summary and Outlook}

The theorem formulated by H. A. Jahn and E. Teller [3] namely that "All non-linear nuclear configurations are unstable for an orbitally degenerate electronic state" and reformulated by Jahn [4] "A configuration of a polyatomic molecule for an electronic state having orbital degeneracy cannot be stable with respect to all displacements of the nuclei unless in the original configuration the nuclei all lie on a straight line" does not contain explicit indications on the (equilibrium) nuclear configuration nor on the chemical reasons or the mechanisms for the spontaneous distortion of the nuclear framework. The manifestations of the theorem are much more diverse than the simplicity of the theorem suggests. As is often the case in molecular physics, each molecule represents a special case. 
In molecules with a high degree of permutational symmetry such as those considered in the present article, several equivalent distorted minimum energy structures result from the Jahn-Teller effect, often connected by a network of tunneling paths and mechanisms. When the energy barriers separating the minimum energy structures are low, the Jahn-Teller effect results in a high fluxionality of the molecule. The corresponding quantum level structures are complex and cannot be measured, analyzed or predicted with the standard methods used for rigid molecules. Experimentally, the use of double-resonance methods, which enable one to group the transitions observed in a spectrum according to the nuclear spin symmetry, greatly facilitate initial assignments. Photoelectron spectroscopy offers distinctive advantages for the study of molecular cations subject to the JT effect. Many vibrational states are accessible, particularly those associated with the JT active modes, and high-resolution methods such as PFI-ZEKE photoelectron spectroscopy give access to their rotational energy level structure. Theoretically, the point groups adequate for rigid molecules can no longer be used to classify the quantum levels and have to be replaced by the molecular symmetry groups [22]. The quantum level structure resulting from the tunneling dynamics can be qualitatively predicted by correlating the point group of the distorted structure to the molecular symmetry group of the fluxional molecule. In conjunction with ab initio quantum chemical calculations, this methodology permits the prediction and classification of the complete rovibronic structure.

Isotopic substitutions are an essential tool in studies of the tunneling dynamics of highly symmetric molecules. They allow one to reduce the permutational symmetry and remove the energetic equivalence of potential energy minima without affecting the nature of the electronic potential energy surfaces. Isotopic substitution enables one to change the zero-point energies of otherwise equivalent structures and to investigate the consequences of the reduced degree of degeneracy on the dynamics of the molecule. In particular we have shown that it can suppress some tunneling paths at low energies, thereby inducing rigidity of the nuclear framework and giving rise to unusual forms of isomerism and chirality.

This review concentrated on two fundamental cations, the methane cation and the cyclopentadienyl cation, which are representative examples of the diversity of spectral, structural and dynamical manifestations of the JT effect in highly symmetric molecules. The common aspect of the JT effect in both cations is that it leads to a partial localization of the electronic wave functions and to a delocalization of the nuclear wave functions over several equivalent configurations. We have determined how these molecular cations distort and have rationalized the distortions using chemical intuition. The methane cation distorts to a structure resembling a 
van der Waals complex of $\mathrm{CH}_{2}^{+}$and $\mathrm{H}_{2}$, which can be attributed to the low ionization energy of $\mathrm{CH}_{2}$ and the strength of the bond of $\mathrm{H}_{2}$. The cyclopentadienyl cation in its lowest singlet state distorts to dienylic and allylic structures that permit the localization of electrons and avoid the delocalized antiaromatic structure.

In future, as theoretical and computational tools will improve, the treatment of an increasing number of degrees of freedom will become possible using highly accurate ab initio quantum chemical calculations and numerically exact computations of vibrational energy levels. Experimentally the measurements of spectra at higher resolution would be desirable, particularly in view of observing spin-orbit and spin-rotational structures and so far unobserved tunneling splittings, which would allow an even more precise characterization of the nuclear structure and dynamics. In particular, direct spectroscopic measurements in the microwave and IR ranges would ideally complement the information obtained by photoelectron spectroscopy.

The JT effect and its consequences on the rovibronic quantum level structure and dynamics of larger highly symmetric molecules such as symmetrically substituted transition metals or $\mathrm{C}_{60}^{+}$remain largely uncharacterized. Obtaining information on these systems constitutes an immense challenge, both theoretically and experimentally, and will hopefully continue to stimulate experimental and theoretical progress.

\section{biographical sketches}

add photographs

Hans Jakob Wörner was born in Freiburg i. Br., Germany, in 1981. He studied chemistry at EPF Lausanne and ETH Zürich from which he graduated in 2003 with a diploma in chemistry. He then started a Ph.D. thesis under the guidance of Prof. F. Merkt on high-resolution photoionization and photoelectron spectroscopy that he completed in 2007. His research focussed on non-Born-Oppenheimer effects in molecular cations for which he obtained the ETH medal for outstanding dissertations. He then worked as a postdoctoral researcher in the group of Dr. Ch. Jungen at the Université de Paris-Sud, Orsay, on the theoretical description of molecular Rydberg states by multichannel quantum defect theory. In 2007 he joined the group of Prof. P. Corkum at NRC in Ottawa, Canada, where he has worked since on new spectroscopic methods with attosecond time resolution.

Frédéric Merkt received his degree in chemistry in 1988 from ETH Zurich and his Ph.D. in 1992 from Cambridge University, England, for work on the generation and spectroscopic 
application of VUV laser radiation carried out under the guidance of Prof. T. P. Softley. He then worked as a postdoctoral researcher in the group of Dr. P. M. Guyon at the Université de Paris-Sud, Orsay (1992) and in the group of Prof. R. N. Zare at Stanford University (19931994). Before returning to ETH Zurich as assistant professor in 1995 he worked at Oxford University where he held a junior research fellowship at St. John's College. Frédéric Merkt has been full Professor of Physical Chemistry since 1999 at ETH Zürich. He has received the Swiss National Latsis Prize and the Alfred Werner Prize of the Swiss Chemical Society in 1999 and the Akademiepreis der Berlin-Brandenburgischen Akademie der Wissenschaften in 2004.

\section{keywords}

keywords: ab initio calculations, fluxionality, isomers, Jahn-Teller effect, photoelectron spectroscopy (all keywords are taken from the list)

\section{for Table of contents}

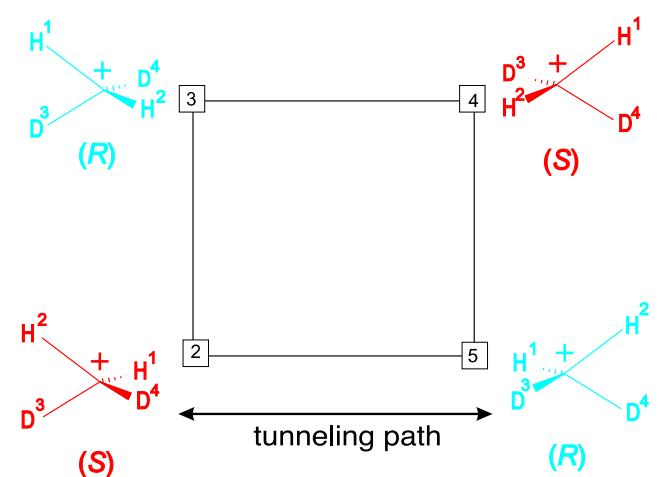

The Jahn-Teller effect in molecules is more complex than a simple geometric distortion. It profoundly alters the electronic and nuclear structures of the molecule, and can result in a high fluxionality. This review shows how rotationally resolved photoelectron spectroscopy in combination with group theory has unraveled precise information about the dynamical structure of the methane cation and the cyclopentadienyl cation. It also demonstrates how the Jahn-Teller effect is linked to antiaromaticity in $\mathrm{C}_{5} \mathrm{H}_{5}^{+}$and induces an unexpected isomerism and chirality in deuterated isotopomers of $\mathrm{CH}_{4}^{+}$. 


\section{Acknowledgements}

We thank Prof. M. Quack for his continued encouraging interest in our research and fruitful discussions. We thank Prof. R. Signorell, Dr. M. Sommavilla, R. van der Veen and Dr. X. Qian, Dr. A. M. Schulenburg, Dr. S. Willitsch and M. Grütter for their contributions to the work summarized in this review article. Over the years we have benefited from many fruitful interactions with Prof. Dr. M. Quack to whom it is a pleasure to dedicate this article on the occasion of his 60th birthday. This work is supported financially by the Swiss National Science Foundation and the ETH Zürich.

\section{References}

[1] I. B. Bersuker, The Jahn-Teller effect. Cambridge University Press, Cambridge, UK 2006.

[2] W. Domcke, D. R. Yarkony, H. Köppel, editors, Conical intersections: Electronic structure, dynamics and spectroscopy, volume 15 of Adv. Ser. in Phys. Chem. World Scientific, Singapore 2004.

[3] H. A. Jahn, E. Teller, Proc. R. Soc. London Ser. A 1937, 161, 220.

[4] H. A. Jahn, Proc. R. Soc. London Ser. A 1938, 164, 117.

[5] H. C. Longuet-Higgins, U. Öpik, M. H. L. Pryce, R. A. Sack, Proc. R. Soc. London Ser. A $1958,244,1$.

[6] H. C. Longuet-Higgins, Advances in Spectroscopy, volume II. Interscience Publishers, New York, London 1961.

[7] I. B. Bersuker, Sov. Phys. JETP 1963, 16, 933.

[8] U. Öpik, M. H. L. Pryce, Proc. R. Soc. London Ser. A 1957, 238, 425.

[9] H. von Busch, V. Dev, H.-A. Eckel, S. Kasahara, J. Wang, W. Demtröder, P. Sebald, W. Meyer, Phys. Rev. Lett. 1998, 81, 4584. see erratum in Phys. Rev. Lett. 82, 3560 (1999).

[10] R. Meiswinkel, H. Köppel, Chem. Phys. 1990, 144, 117.

[11] W. E. Ernst, S. Rakowsky, Phys. Rev. Lett. 1995, 74, 58. 
[12] R. Lindner, K. Müller-Dethlefs, E. Wedum, K. Haber, E. R. Grant, Science 1996, 271, 1698.

[13] M. Ford, R. Lindner, K. Müller-Dethlefs, Mol. Phys. 2003, 101, 705.

[14] H. Köppel, L. S. Cederbaum, W. Domcke, S. S. Shaik, Angew. Chem. (int. ed. engl.) 1983, 22, 210.

[15] H. Köppel, W. Domcke, L. S. Cederbaum, Adv. Chem. Phys. 1984, 57, 59.

[16] J. W. Zwanziger, E. R. Grant, J. Chem. Phys. 1987, 87, 2954.

[17] T. A. Barckholtz, T. A. Miller, Int. Rev. Phys. Chem. 1998, 17, 435.

[18] B. E. Applegate, T. A. Barckholtz, T. A. Miller, Chem. Soc. Rev. 2003, 32, 38.

[19] C. C. Chancey, M. C. M. O'Brien, The Jahn-Teller effect in $C_{60}$ and other icosahedral complexes. Princeton University Press 1997.

[20] K. Müller-Dethlefs, E. W. Schlag, Ann. Rev. Phys. Chem. 1991, 42, 109.

[21] K. Müller-Dethlefs, E. W. Schlag, Angew. Chem. (int. ed. engl.) 1998, 37, 1346.

[22] P. R. Bunker, P. Jensen, Molecular Symmetry and Spectroscopy. NRC Research Press, Ottawa, 2nd. Aufl. 1998.

[23] H. Köppel, L. S. Cederbaum, W. Domcke, Chem. Phys. Lett. 1984, 110, 469.

[24] I. B. Bersuker, V. Z. Polinger, Sov. Phys. JETP 1974, 39, 1023.

[25] A. Bohm, A. Mostafazadeh, H. Koizumi, Q. Niu, J. Zwanziger, The geometric phase in quantum systems: Foundations, Mathematical Concepts and Applications in Molecular and Condensed Matter Physics. Springer-Verlag, Heidelberg 2003.

[26] J. K. Cullum, R. A. Willoughby, Lanczos Algorithms for Large Symmetric Eigenvalue Computations. Birkhauser, Boston 1985.

[27] M. J. Paterson, M. J. Bearpark, M. A. Robb, L. Blancafort, G. A. Worth, Phys. Chem. Chem. Phys. 2008, 7, 2100.

[28] G. Herzberg, H. C. Longuet-Higgins, Diss. Farad. Soc. 1963, 35, 77.

[29] H. J. Wörner, R. van der Veen, F. Merkt, Phys. Rev. Lett. 2006, 97, 173003. 
[30] W. T. Borden, Diradicals. John Wiley and Sons, New York 1982.

[31] H. Köppel, L. S. Cederbaum, W. Domcke, J. Chem. Phys. 1988, 89, 2023.

[32] J. K. G. Watson, Mol. Phys. 1999, 96, 1721.

[33] W. T. Borden, E. R. Davidson, J. Am. Chem. Soc. 1979, 101, 3771.

[34] H. J. Wörner, F. Merkt, J. Chem. Phys. 2007, 127, 034303.

[35] M. N. Paddon-Row, D. J. Fox, J. A. Pople, K. N. Houk, D. W. Pratt, J. Am. Chem. Soc. $1985,10 \%, 7696$.

[36] R. F. Frey, E. R. Davidson, J. Chem. Phys. 1988, 88, 1775.

[37] R. Signorell, F. Merkt, Faraday Discuss. 2000, 115, 205.

[38] M. Quack, Angew. Chem. (int. ed. engl.) 1989, 28, 571.

[39] M. Quack, J. Stohner, M. Willeke, Ann. Rev. Phys. Chem. 2008, 59, 741.

[40] M. S. Reeves, E. R. Davidson, J. Chem. Phys. 1991, 95, 6551.

[41] H. J. Wörner, X. Qian, F. Merkt, J. Chem. Phys. 2007, 126, 144305.

[42] G. Herzberg, Molecular Spectra and Molecular Structure, Volume II, Infrared and Raman Spectra of Polyatomic Molecules. Krieger Publishing Company, Malabar 1991.

[43] F. Merkt, Ann. Rev. Phys. Chem. 1997, 48, 675.

[44] E. W. Schlag, ZEKE spectroscopy. Cambridge University Press, Cambridge, UK 1998.

[45] U. Hollenstein, R. Seiler, H. Schmutz, M. Andrist, F. Merkt, J. Chem. Phys. 2001, 115, 5461.

[46] D. E. Cooper, C. M. Klimack, J. E. Wessels, Phys. Rev. Lett. 1981, 46, 324.

[47] B. Bobin, J. Physique 1972, 33, 345.

[48] S. Willitsch, J. M. Dyke, F. Merkt, Helv. Chim. Acta 2003, 86, 1152.

[49] H. J. Wörner, F. Merkt, Angew. Chem. (int. ed. engl.) 2006, 45, 293.

[50] M. Saunders, R. Berger, A. Jaffe, J. M. McBride, J. O'Neill, R. Breslow, J. M. Hoffmann, C. Perchonock, E. Wassermann, R. S. Hutton, V. J. Kuck, J. Am. Chem. Soc. 1973, 95, 3017. 
[51] T. Bally, S. Masamune, Tetrahedron 1980, 36, 343.

[52] G. Maier, Angew. Chem. (int. ed. engl.) 1988, 27, 309.

[53] R. Signorell, F. Merkt, J. Chem. Phys. 1999, 110, 2309.

[54] M. Quack, Mol. Phys. 1977, 34, 477.

[55] M. Hippler, M. Quack, Chem. Phys. Lett. 1999, 314, 273.

[56] S. Willitsch, L. L. Imbach, F. Merkt, J. Chem. Phys. 2002, 117, 1939.

[57] A. Carrington, H. Longuet-Higgins, R. Moss, P. Todd, Mol. Phys. 1965, 9, 187.

[58] R. G. Lawler, J. R. Bolton, G. K. Fraenkel, T. H. Brown, J. Am. Chem. Soc. 1964, 86, 520.

[59] B. Scharf, R. Vitenberg, B. Katz, Y. B. Band, J. Chem. Phys. 1982, 77, 2226.

[60] L. Yu, D. W. Cullin, J. M. Williamson, T. A. Miller, J. Chem. Phys. 1993, 98, 2682.

[61] H. J. Wörner, F. Merkt, J. Chem. Phys. 2007, 126, 154304.

[62] R. Signorell, M. Sommavilla, F. Merkt, Chem. Phys. Lett. 1999, 312, 139.

[63] M. Quack, Nova Acta Leopoldina NF 1999, 81, 1.

[64] R. Berger, G. Laubender, M. Quack, A. Sieben, J. Stohner, M. Willeke, Angew. Chem. Intl. Ed. 2005, 44, 3623.

[65] L. Wang, J. E. Pollard, Y. T. Lee, D. A. Shirley, J. Chem. Phys. 1987, 86, 3216.

[66] S. Willitsch, U. Hollenstein, F. Merkt, J. Chem. Phys. 2004, 120, 1761.

[67] A. M. Schulenburg, Diss. ETH Nr. 18024, Dissertation, Eidgenössische Technische Hochschule ETH Zürich, 2008.

[68] B. Fehrensen, D. Luckhaus, M. Quack, Chem. Phys. Lett. 1999, 300, 312.

[69] M. Gottselig, D. Luckhaus, M. Quack, J. Stohner, M. Willeke, Helv. Chim. Acta 2001, 84, 1846.

[70] R. Berger, M. Gottselig, M. Quack, M. Willeke, Angew. Chem. Intl. Ed. 2001, 40, 4195.

[71] M. Gottselig, M. Quack, J. Chem. Phys. 2005, 123, 84305. 
[72] M. J. M. Pepper, I. Shavitt, P. von Ragué Schleyer, M. N. Glukhovtsev, R. Janoschek, M. Quack, J. Comp. Chem. 1995, 16, 207.

[73] M. J. Frisch, G. W. Trucks, H. B. Schlegel, G. E. Scuseria, M. A. Robb, J. R. Cheeseman, J. A. Montgomery, Jr., T. Vreven, K. N. Kudin, J. C. Burant, J. M. Millam, S. S. Iyengar, J. Tomasi, V. Barone, B. Mennucci, M. Cossi, G. Scalmani, N. Rega, G. A. Petersson, H. Nakatsuji, M. Hada, M. Ehara, K. Toyota, R. Fukuda, J. Hasegawa, M. Ishida, T. Nakajima, Y. Honda, O. Kitao, H. Nakai, M. Klene, X. Li, J. E. Knox, H. P. Hratchian, J. B. Cross, V. Bakken, C. Adamo, J. Jaramillo, R. Gomperts, R. E. Stratmann, O. Yazyev, A. J. Austin, R. Cammi, C. Pomelli, J. W. Ochterski, P. Y. Ayala, K. Morokuma, G. A. Voth, P. Salvador, J. J. Dannenberg, V. G. Zakrzewski, S. Dapprich, A. D. Daniels, M. C. Strain, O. Farkas, D. K. Malick, A. D. Rabuck, K. Raghavachari, J. B. Foresman, J. V. Ortiz, Q. Cui, A. G. Baboul, S. Clifford, J. Cioslowski, B. B. Stefanov, G. Liu, A. Liashenko, P. Piskorz, I. Komaromi, R. L. Martin, D. J. Fox, T. Keith, M. A. Al-Laham, C. Y. Peng, A. Nanayakkara, M. Challacombe, P. M. W. Gill, B. Johnson, W. Chen, M. W. Wong, C. Gonzalez, J. A. Pople. Gaussian 03, Revision C.02. Gaussian, Inc., Wallingford, CT, 2004 .

[74] A. Garg, Am. J. Phys. 2000, 68, 430.

[75] J. T. Hougen. Methane Symmetry operation, MTP International Reviews of Science, Physical Chemistry. In Methane Symmetry operation, MTP International Reviews of Science, Physical Chemistry, D. A. Ramsay, editor, volume 3. Butterworth, London 1976.

[76] A. Olah, G. Rasul, Acc. Chem. Res. 1997, 30, 245.

[77] E. Herbst, J. Phys. Chem. A 2005, 109, 4017.

[78] E. T. White, J. Tang, T. Oka, Science 1999, 284, 135.

[79] O. Asvany, P. Kumar, B. Redlich, I. Hegemann, S. Schlemmer, D. Marx, Science 2005, $309,1219$.

[80] X. Huang, A. B. McCoy, J. M. Bowman, L. M. Johnson, C. Savage, F. Dong, D. J. Nesbitt, Science 2006, 311, 60.

[81] X.-G. Wang, T. Carrington Jr., J. Chem. Phys. 2008, 129, 234102.

[82] A. Kormornicki, D. A. Dixon, J. Chem. Phys. 1987, 86, 5625. 
[83] W. Klopper, W. Kutzelnigg, J. Phys. Chem. 1990, 94, 5625.

[84] P. R. Schreiner, S.-J. Kim, H. F. Schafer III, P. von Ragué Schleyer, J. Chem. Phys. 1993, 99, 3716.

[85] P. R. Schreiner, Angew. Chem. Int. Ed. 2000, 39, 3239.

[86] A. Brown, A. B. McCoy, B. J. Braams, Z. Jin, J. M. Bowman, J. Chem. Phys. 2004, 121, 4105.

[87] Z. Jin, B. J. Braams, J. M. Bowman, J. Phys. Chem. A 2006, 110, 1569.

[88] P. R. Bunker, B. Ostojić, S. Yurchenko, J. Mol. Struc. 2004, 695-696, 253.

[89] A. B. McCoy, B. J. Braams, A. Brown, X. Huang, Z. Jin, J. M. Bowman, J. Phys. Chem. A 2004, 108, 4991.

[90] L. M. Johnson, A. B. McCoy, J. Phys. Chem. A 2006, 810, 8213.

[91] X. Huang, L. M. Johnson, J. M. Bowman, A. B. McCoy, J. Am. Chem. Soc. 2006, 128, 3478 . 


\section{Figure Captions}

Fig. 1. Frost-Musulin diagram of the lowest-lying electronic configuration of the cyclopentadienyl cation (left-hand side) and energetic ordering of the corresponding electronic states in $\mathrm{D}_{5 \mathrm{~h}}$ symmetry (right-hand side). $K_{23}$ represents the exchange integral (see text; Figure adapted from Ref. [34]).

Fig. 2. Adiabatic potential energy surfaces resulting from a linear Jahn-Teller effect in a doubly degenerate electronic state induced by a single doubly degenerate vibrational mode (see Eq. (18)). The parameters used for the figure are $\omega_{\mathrm{JT}}=800 \mathrm{~cm}^{-1}$ and $g=500 \mathrm{~cm}^{-1}$.

Fig. 3. Adiabatic potential energy surfaces resulting from a linear Pseudo-Jahn-Teller effect between a lower-lying doubly degenerate electronic state (E) and a higher-lying non-degenerate state (A) induced by a single doubly degenerate vibrational mode (see Eq. (19)). The parameters used for the figure are $\omega_{\mathrm{PJT}}=800 \mathrm{~cm}^{-1}, V_{\mathrm{A}}(0)-V_{\mathrm{E}}(0)=5600 \mathrm{~cm}^{-1}$ and $\lambda=2400 \mathrm{~cm}^{-1}$.

Fig. 4. Adiabatic potential energy surfaces arising from the linear Jahn-Teller effect (left panel) and the Pseudo-Jahn-Teller effect in the ${ }^{1} \mathrm{E}_{2}^{\prime}$ and ${ }^{1} \mathrm{~A}_{1}^{\prime}$ states (right panel). The axes are chosen to be the $\mathrm{x}$ components of the effective $\mathrm{e}_{1}^{\prime}$ and $\mathrm{e}_{2}^{\prime}$ modes that connect the $\mathrm{D}_{5 \mathrm{~h}}$ geometry point (indicated by the vertical line) to the respective minima. Both modes preserve $\mathrm{C}_{2 \mathrm{v}}$ symmetry which enables the attribution of electronic symmetries to each curve. Electronic symmetries in the $\mathrm{D}_{5 \mathrm{~h}}$ group are given at the symmetric point.

Fig. 5. a) Schematic representation of the minimum energy pseudorotation path in the lowest singlet state. A distortion along the $\mathrm{e}_{2 \mathrm{x}}^{\prime}$ dimension preserves $\mathrm{C}_{2 \mathrm{v}}$ symmetry and takes the molecule to either the allylic or dienylic structure. If vibronic coupling terms of third and higher orders are neglected, the displayed minimum energy path is isoenergetic. b) Electronic configurations of the $\tilde{a}^{+1} \mathrm{E}_{2}^{\prime}$ state at $\mathrm{D}_{5 \mathrm{~h}}$ symmetry (middle) and of the distorted dienylic and allylic structures (left and right, respectively). The ordering of the $a_{2}$ and $b_{1}$ molecular orbitals originating from the degenerate $\mathrm{e}_{1}^{\prime \prime}$ orbital is opposite in the dienylic and allylic structures (adapted from Ref. [34]).

Fig. 6. Equilibrium geometry of $\mathrm{CH}_{4}^{+}$, structure of its highest lying singly occupied molecular orbital at the global minimum of the potential energy surface at the UMP2/cc-pVDZ level of $a b$ initio theory and principal axes of inertia. The structure has $\mathrm{C}_{2 \mathrm{v}}$ geometry and the $\mathrm{HCH}$ bond angles and $\mathrm{C}-\mathrm{H}$ bond distances are indicated.

Fig. 7. Schematic representation of the three potential energy surfaces of the methane cation arising from the Jahn-Teller effect in its ${ }^{2} \mathrm{~F}_{2}$ ground state. The vertical axis represents the 
electronic potential energy, whereas the horizontal axis represents effective displacement coordinates the symmetries of which are indicated. The structures corresponding to the represented stationary points and their point group are indicated below the graph.

Fig. 8. Topological representation of the connectivity of the six equivalent minimum energy structures of $\mathrm{C}_{2 \mathrm{v}}$ symmetry of $\mathrm{CH}_{4}^{+}$. The vertices correspond to the $\mathrm{C}_{2 \mathrm{v}}$ minimum energy geometries and the edges to the equivalent pseudorotation-tunneling paths connecting the minima via low-lying $\mathrm{C}_{\mathrm{s}}$ transition states. Four faces of the octahedron correspond to a $\mathrm{C}_{3 \mathrm{v}}$ geometry with a degenerate ground state (structure $\mathrm{C}_{3 \mathrm{v}}$ (II) of Fig. 7 , marked with a dot) and the other four to a $\mathrm{C}_{3 \mathrm{v}}$ geometry with a nondegenerate ground state (structure $\mathrm{C}_{3 \mathrm{v}}(\mathrm{I}$ ) of Fig. 7). The adiabatic electronic wave function $|n\rangle$ is indicated below each structure as a linear combination of the three diabatic basis functions $(|\xi\rangle,|\eta\rangle,|\zeta\rangle)$ defined at the $\mathrm{T}_{\mathrm{d}}$ geometry.

Fig. 9. One- and two-photon excitation schemes used to record photoionization and PFI-ZEKE photoelectron spectra of the cyclopentadienyl radical. The positions of the low-lying electronic states of the cyclopentadienyl radical and cation are represented on the left-hand side of the figure by the horizontal lines next to the corresponding spectroscopic and symmetry labels. The main electronic configuration of the $\pi$ molecular orbitals from which the electronic states derive are represented schematically in the central column. The right-hand side represents schematic cuts through the potential energy surface of the electronic states along nuclear displacements of $\mathrm{e}_{2}^{\prime}$ symmetry (adapted from Ref. [34]).

Fig. 10. Two-photon resonant PFI-ZEKE photoelectron spectra recorded via the vibrationless level of the $\tilde{\mathrm{A}}^{2} \mathrm{~A}_{2}^{\prime \prime}$ state of $\mathrm{C}_{5} \mathrm{H}_{5}$ (panel a) and $\mathrm{C}_{5} \mathrm{D}_{5}$ (panel b). Vertical arrows mark the positions of the adiabatic ionization thresholds corresponding to the formation of the $\tilde{\mathrm{X}}^{+3} \mathrm{~A}_{2}^{\prime}$ and lower component of the $\tilde{a}^{+1} \mathrm{E}_{2}^{\prime}$ states, respectively. The horizontal axis corresponds to the sum of the wave numbers of both lasers. Excited vibrational levels of the $\tilde{\mathrm{X}}^{+3} \mathrm{~A}_{2}^{\prime}$ state are assigned in harmonic notation whereas the pseudorotational levels of the lower component of the $\tilde{\mathrm{a}}^{+1} \mathrm{E}_{2}^{\prime}$ state are assigned in terms of the vibronic angular momentum quantum number $j$ (adapted from Ref. [34]).

Fig. 11. Top trace: Single-photon PFI-ZEKE photoelectron spectrum of $\mathrm{CH}_{4}$ in the region of the adiabatic ionization threshold obtained using electric field pulses of $+86 \mathrm{mV} \mathrm{cm}^{-1}$ and -138 $\mathrm{mV} \mathrm{cm}{ }^{-1}$. Lower traces: two-photon IR+VUV PFI-ZEKE PE spectra recorded via selected rotational levels of the $\nu_{3}$ fundamental using electric field pulses of $+17 \mathrm{mV} \mathrm{cm}^{-1}$ and $-860 \mathrm{mV}$ $\mathrm{cm}^{-1}$. The rotational angular momentum quantum number $J$ of the intermediate levels and their rovibronic symmetries are indicated above the spectra. The letters A, E and F correspond 
to the experimentally assigned nuclear spin symmetries $\left(\mathrm{A}_{1}, \mathrm{E}\right.$ or $\mathrm{F}_{2}$; Figure adapted from Ref. [41]).

Fig. 12. Comparison of the experimentally determined level structure of $\mathrm{CH}_{4}^{+}$(a) with the eigenvalues of a tunnel-rotation Hamiltonian (see Ref. [29]). The wave number scale is defined with respect to the ground state of $\mathrm{CH}_{4}$ (adapted from Ref. [41]).

Fig. 13. Tunneling levels of $\mathrm{CH}_{3} \mathrm{D}^{+}$with zero total angular momentum (full lines). The quantities $\sigma$ and $\sigma^{\prime}$ represent the tunneling integrals for the isomers $\mathrm{CH}_{3} \mathrm{D}_{s}^{+}$and $\mathrm{CH}_{3} \mathrm{D}_{\ell}^{+}$, respectively, ZPED stands for the zero-point energy difference between these isomers and $\Delta$ describes the zero-point energy difference in the absence of tunneling (adapted from Ref. [61]).

Fig. 14. PFI-ZEKE photoelectron spectrum (full line) and photoionization spectrum (dashed line) of $\mathrm{CH}_{3} \mathrm{D}$. The PFI-ZEKE photoelectron spectrum was recorded using a sequence of pulsed electric fields of $+35 \mathrm{mV} \mathrm{cm}^{-1}$ and $-130 \mathrm{mV} \mathrm{cm}^{-1}$. The two bands are assigned to the two isomers $\mathrm{CH}_{3} \mathrm{D}_{\ell}^{+}$and $\mathrm{CH}_{3} \mathrm{D}_{s}^{+}$as indicated in the figure.

Fig. 15. Tunneling levels of $\mathrm{CH}_{2} \mathrm{D}_{2}^{+}$with zero total angular momentum (full lines). The quantity $\sigma$ represents the tunneling integral for the isomers $\mathrm{CH}_{\ell} \mathrm{H}_{s} \mathrm{D}_{\ell} \mathrm{D}_{s}^{+} . \Delta$ and $\Delta^{\prime}$ describe the zero-point energy differences in the absence of tunneling.

Fig. 16. PFI-ZEKE photoelectron spectrum of $\mathrm{CH}_{2} \mathrm{D}_{2}$. The PFI-ZEKE photoelectron spectrum was recorded using a sequence of pulsed electric fields of $+35 \mathrm{mV} \mathrm{cm}^{-1}$ and $-160 \mathrm{mV} \mathrm{cm}^{-1}$. The three bands are assigned to the three isomers $\mathrm{CH}_{\ell} \mathrm{H}_{\ell} \mathrm{D}_{s} \mathrm{D}_{s}^{+}, \mathrm{CH}_{\ell} \mathrm{H}_{s} \mathrm{D}_{\ell} \mathrm{D}_{s}^{+}$and $\mathrm{CH}_{s} \mathrm{H}_{s} \mathrm{D}_{\ell} \mathrm{D}_{\ell}^{+}$, as indicated in the figure.

Fig. 17. Schematic representation of the lowest potential energy surfaces of the ethylene cation $\left(\mathrm{C}_{2} \mathrm{H}_{4}^{+}\right)$. The vertical axis represents the electronic potential energy and the horizontal axis the angle $\theta$ between the two $\mathrm{CH}_{2}$ planes.

Fig. 18. Topological representation of four equivalent minima of the isomer $\mathrm{CH}_{\ell} \mathrm{H}_{s} \mathrm{D}_{\ell} \mathrm{D}_{s}^{+}$of $\mathrm{CH}_{2} \mathrm{D}_{2}^{+}$. The vertices correspond to the $\mathrm{C}_{1}$ minimum energy geometries and the edges to the equivalent pseudorotation-tunneling paths connecting the minima. Neighboring structures are enantiomers (disregarding the numbering) but diametrically opposed structures are identical. The chirality nomenclature used for axially chiral systems has been applied to label the structures.

Fig. 19. Stationary points of the lowest potential energy surface of $\mathrm{CH}_{5}^{+}$. Structure $\mathrm{C}_{\mathrm{s}}(\mathrm{I})$ corresponds to a global electronic potential minimum whereas structures $\mathrm{C}_{\mathrm{s}}(\mathrm{II})$ and $\mathrm{C}_{2 \mathrm{v}}$ correspond to low-lying saddle-points. The electronic potential energy differences taken from Ref. [86]are indicated. 


\section{Table Captions}

Table I. Vibrational symmetries of Jahn-Teller active modes in a state of electronic symmetry $\mathrm{E}_{2}^{\prime}$ and Pseudo-Jahn-Teller active modes coupling a state of electronic symmetry $\mathrm{E}_{2}^{\prime}$ with a state of symmetry $A_{1}^{\prime}$ for a molecule of symmetry $D_{5 h}(M)$ up to third order.

Table II. Vibrational symmetries of Jahn-Teller active modes in a state of electronic symmetry $\mathrm{F}_{2}$ for a molecule of symmetry $\mathrm{T}_{\mathrm{d}}(\mathrm{M})$ up to third order.

Table III. Correlation table of the rovibronic symmetries from the $T_{d}(M)$ to the $S_{4}^{*}$ molecular symmetry group including nuclear spin statistical weights for ${ }^{12} \mathrm{CH}_{4}^{+}$.

Table IV. Same as Table III but with nuclear spin statistical weights for ${ }^{12} \mathrm{CD}_{4}^{+}$. 
Table I:

\begin{tabular}{|c|c|}
\hline \multicolumn{2}{|c|}{$\mathrm{JT}$ activity in $\mathrm{E}_{2}^{\prime}$} \\
\hline order & $\Gamma_{\text {vib }}$ \\
\hline 1 & $\mathrm{e}_{1}^{\prime}$ \\
\hline 2 & $\mathrm{e}_{2}^{\prime}, \mathrm{e}_{2}^{\prime \prime}$ \\
\hline 3 & $\mathrm{e}_{1}^{\prime}, \mathrm{e}_{2}^{\prime}$ \\
\hline \multicolumn{2}{|c|}{ PJT activity $\mathrm{E}_{2}^{\prime} \leftrightarrow \mathrm{A}^{\prime}$} \\
\hline order & $\Gamma_{\text {vib }}$ \\
\hline 1 & $\mathrm{e}_{2}^{\prime}$ \\
\hline 2 & $\mathrm{e}_{1}^{\prime}, \mathrm{e}_{1}^{\prime \prime}$ \\
\hline 3 & $\mathrm{e}_{1}^{\prime}, \mathrm{e}_{2}^{\prime}$ \\
\hline
\end{tabular}

Table II:

\begin{tabular}{cc}
\hline \hline \multicolumn{2}{c}{ JT activity in $\mathrm{F}_{2}$} \\
\hline order & $\Gamma_{\text {vib }}$ \\
\hline 1 & e,f $f_{2}$ \\
2 & $e, f_{2}$ \\
3 & e,f $f_{2}$ \\
\hline \hline
\end{tabular}

Table III:

\begin{tabular}{|cc|}
\hline $\mathrm{T}_{d}(\mathrm{M})$ & $\mathrm{S}_{4}^{*}$ \\
\hline$A_{1}(5)$ & $A_{1}^{+}(0) \oplus A_{2}^{-}(5)$ \\
$A_{2}(5)$ & $A_{2}^{+}(5) \oplus A_{1}^{-}(0)$ \\
$E(2)$ & $E^{+}(1) \oplus E^{-}(1)$ \\
$F_{1}(3)$ & $F_{1}^{+}(3) \oplus F_{2}^{-}(0)$ \\
$F_{2}(3)$ & $F_{2}^{+}(0) \oplus F_{1}^{-}(3)$ \\
\hline
\end{tabular}


Table IV:

\begin{tabular}{|cc|}
\hline $\mathrm{T}_{d}(\mathrm{M})$ & $\mathrm{S}_{4}^{*}$ \\
\hline$A_{1}(15)$ & $A_{1}^{+}(15) \oplus A_{2}^{-}(0)$ \\
$A_{2}(15)$ & $A_{2}^{+}(0) \oplus A_{1}^{-}(15)$ \\
$E(12)$ & $E^{+}(6) \oplus E^{-}(6)$ \\
$F_{1}(18)$ & $F_{1}^{+}(3) \oplus F_{2}^{-}(15)$ \\
$F_{2}(18)$ & $F_{2}^{+}(15) \oplus F_{1}^{-}(3)$ \\
\hline
\end{tabular}



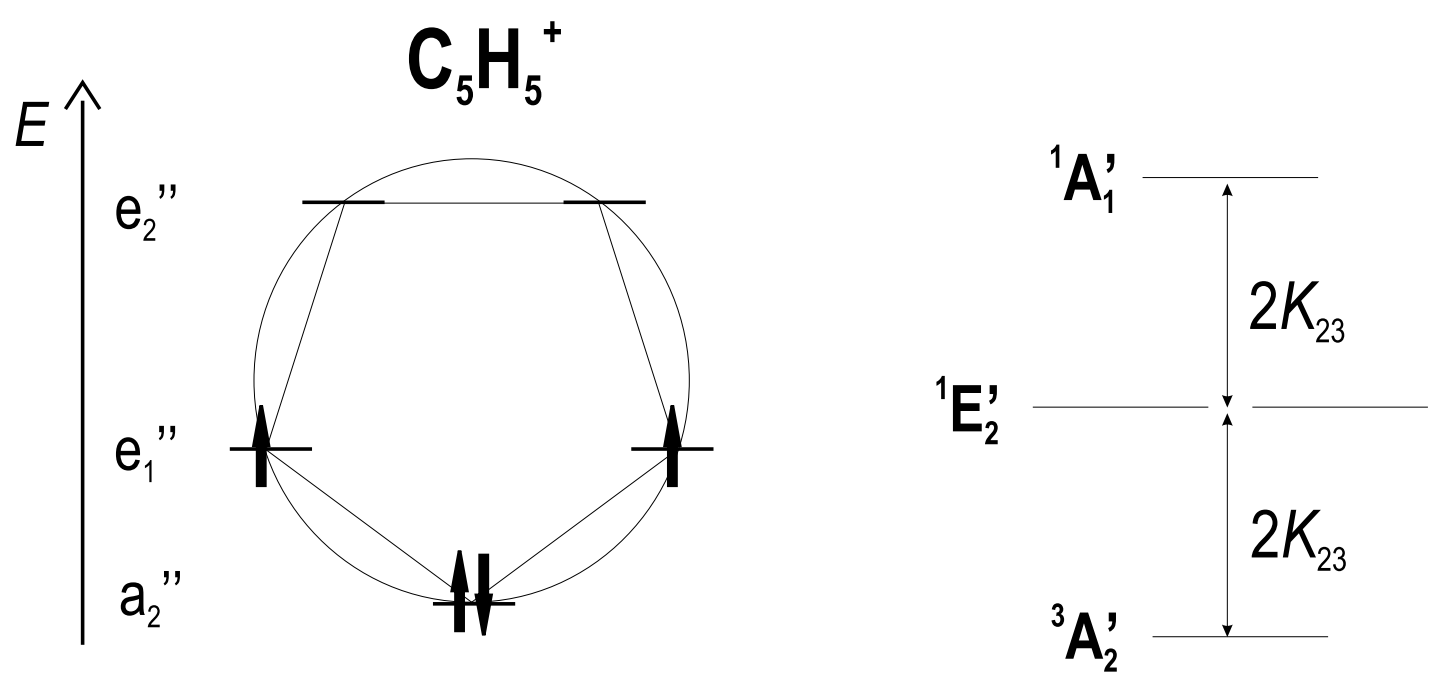

Figure 1: 


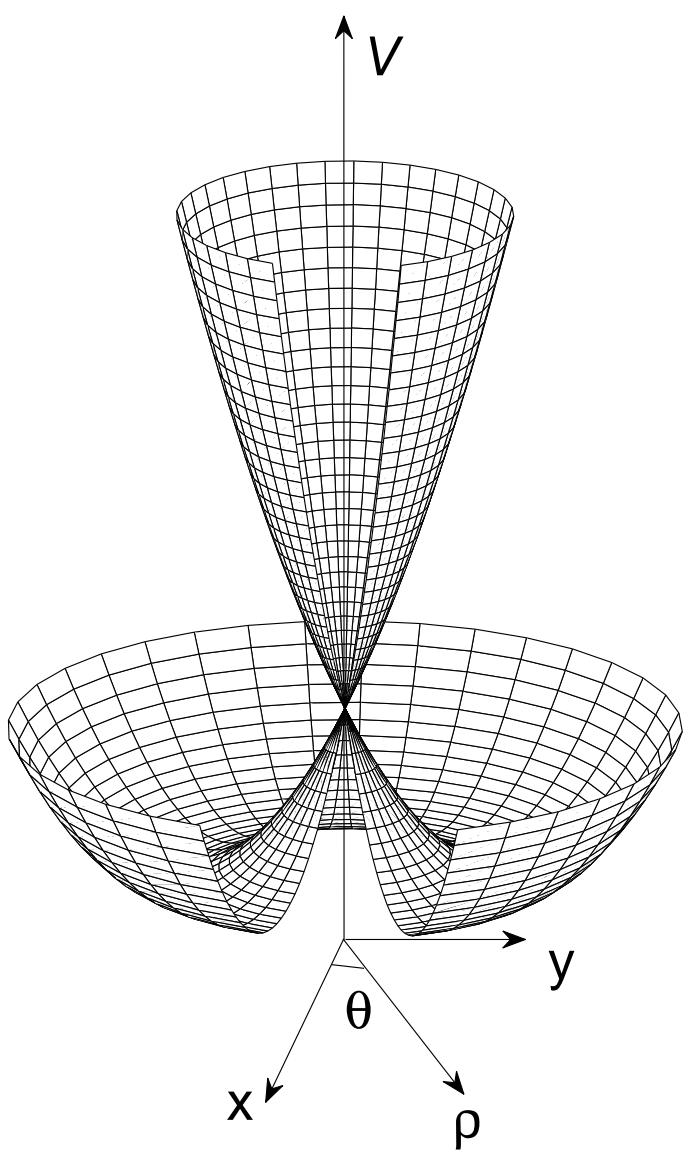

Figure 2: 


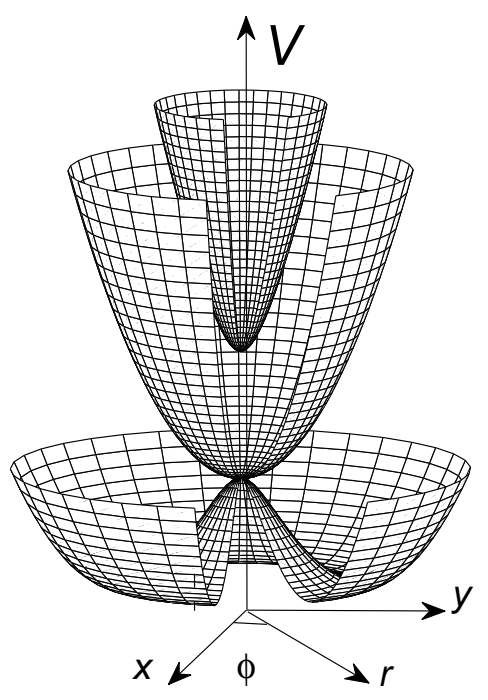

Figure 3: 

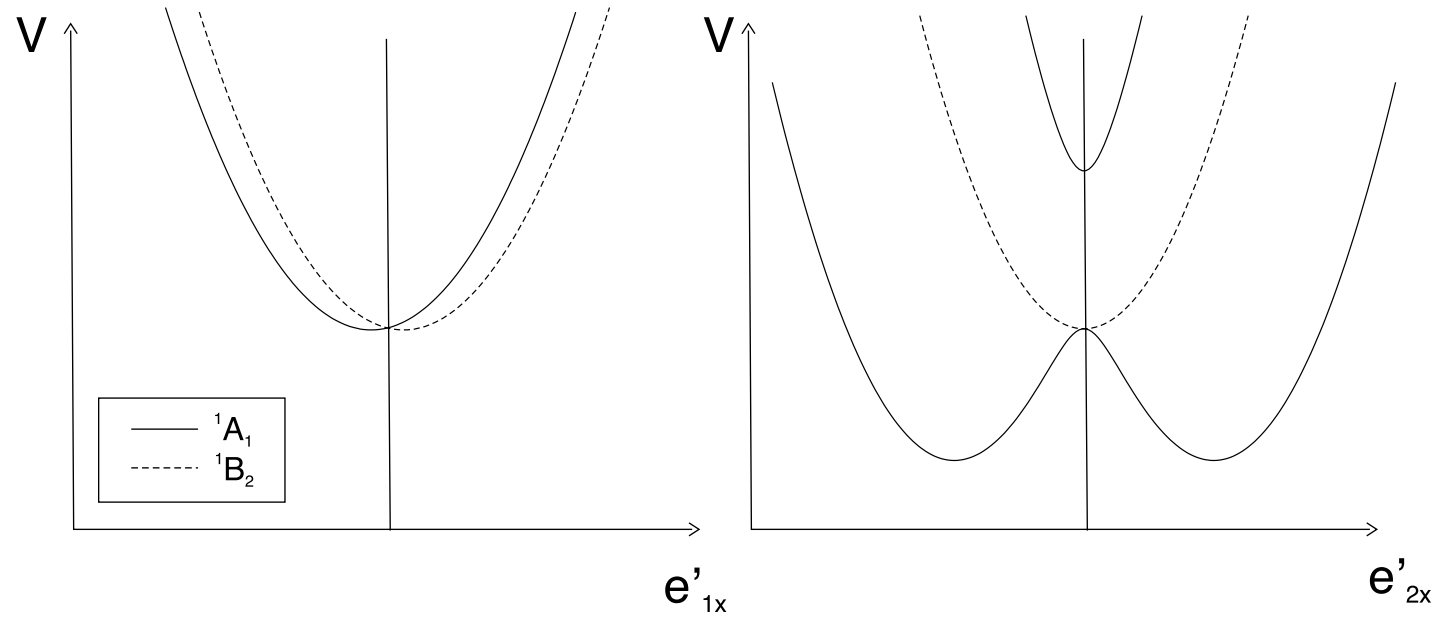

Figure 4: 
a)

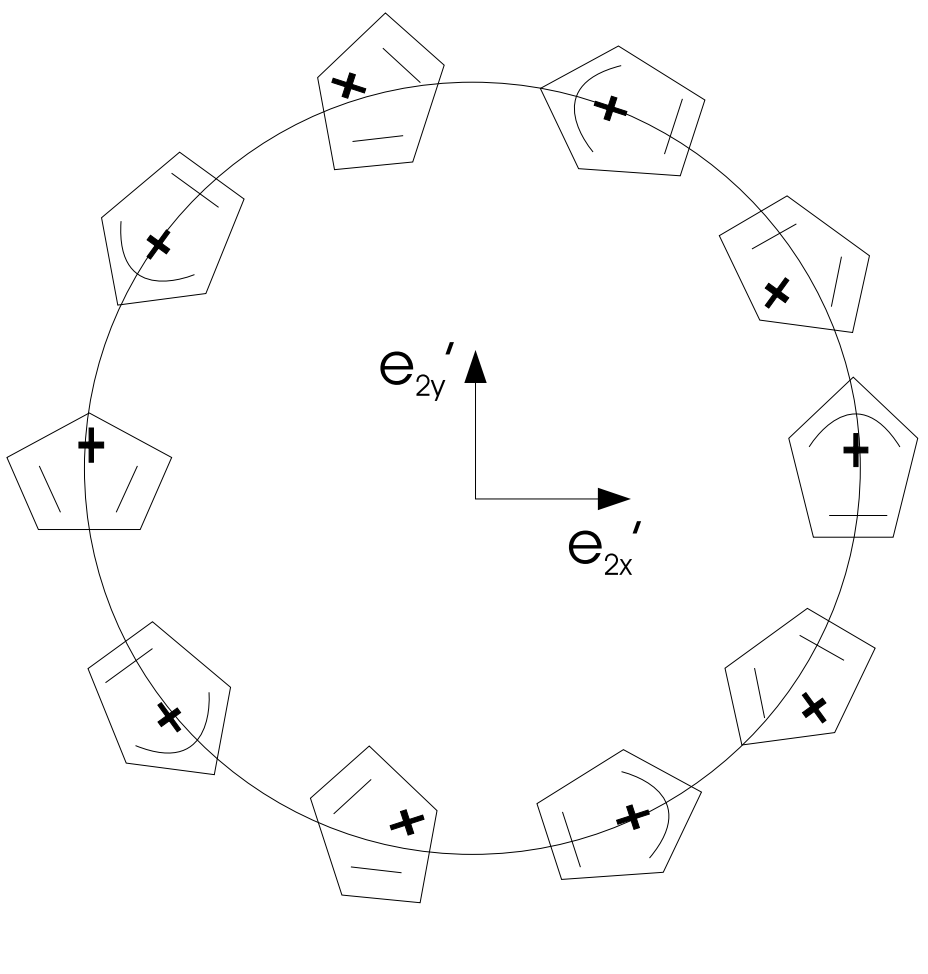

E

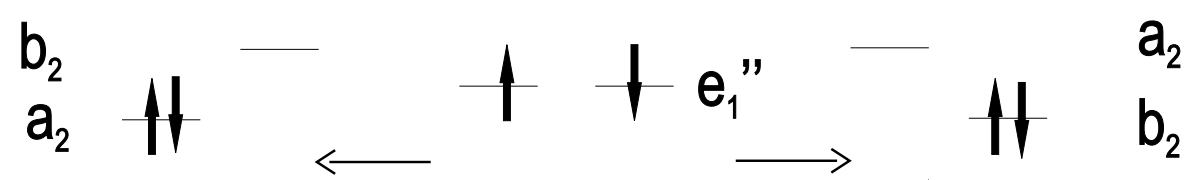

$$
\begin{aligned}
& \text { ft } \\
& + \\
& + \\
& + \\
& { }^{1} \mathrm{~A}_{1} \\
& \text { 'E }{ }^{\prime} \\
& { }^{\prime} \mathrm{A}
\end{aligned}
$$

Figure 5: 


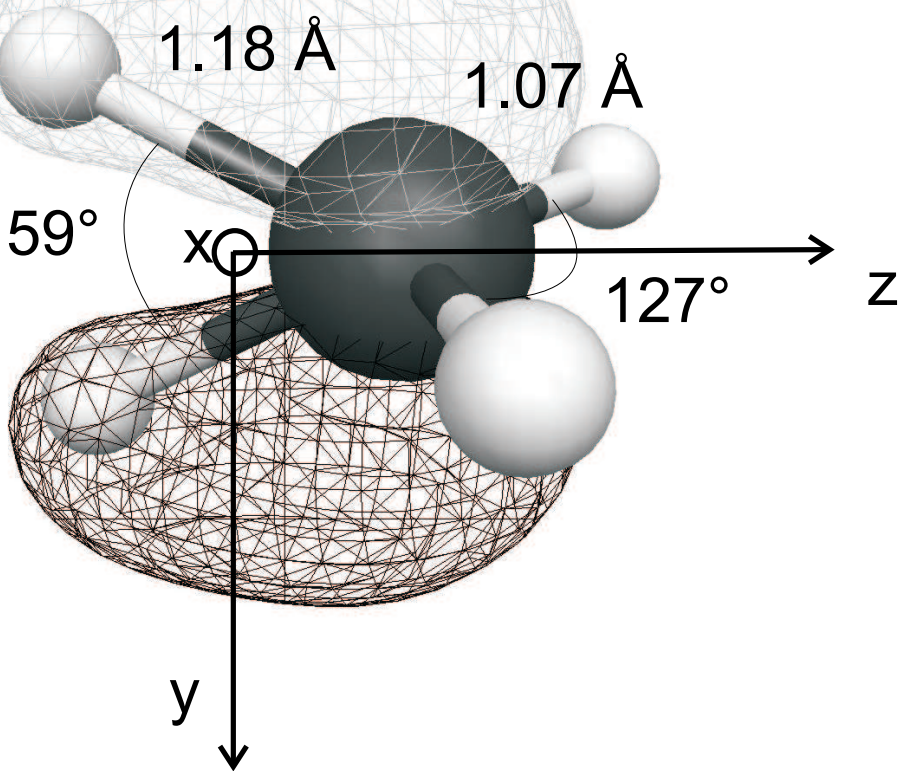

Figure 6: 


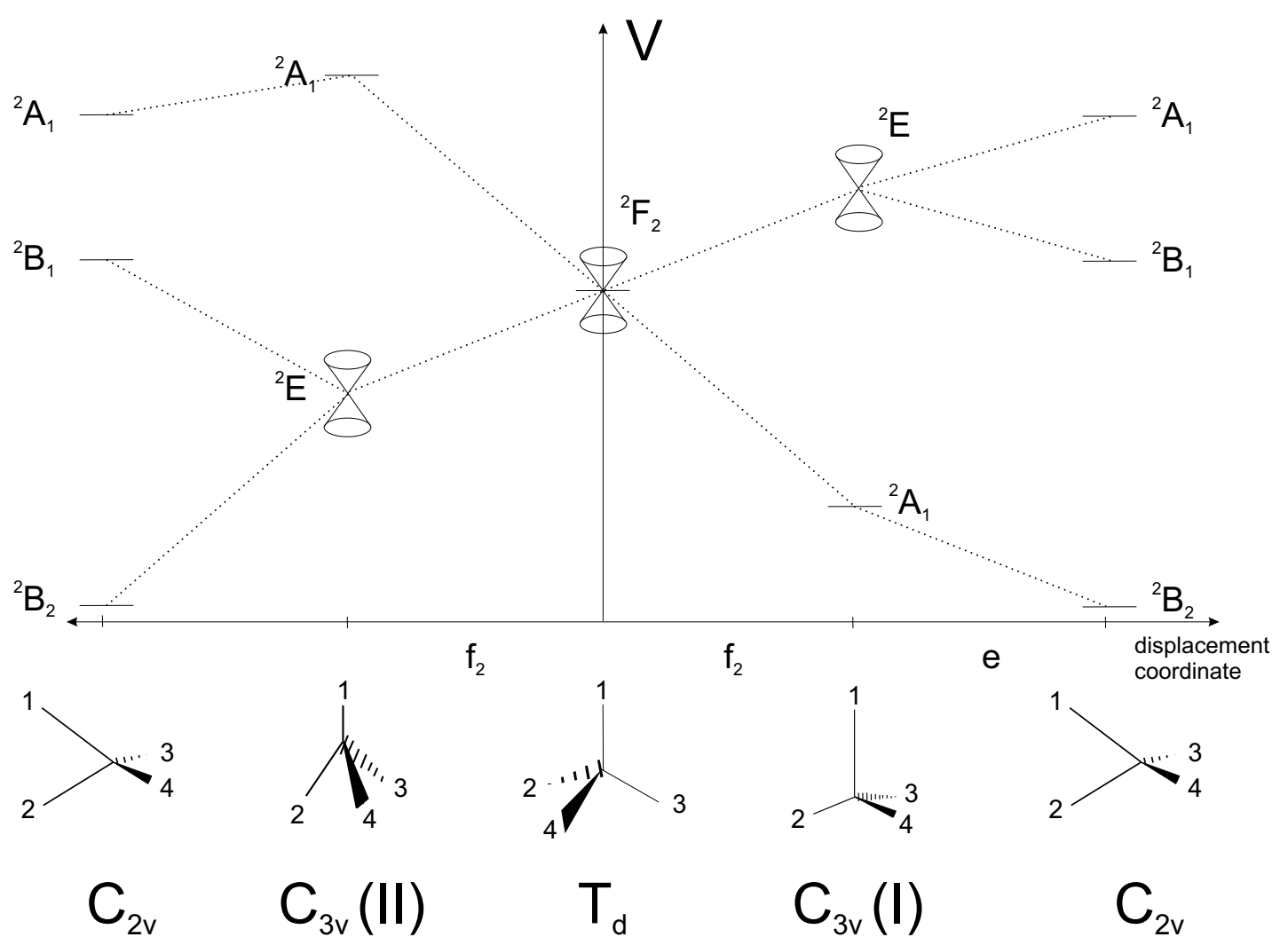

Figure 7: 


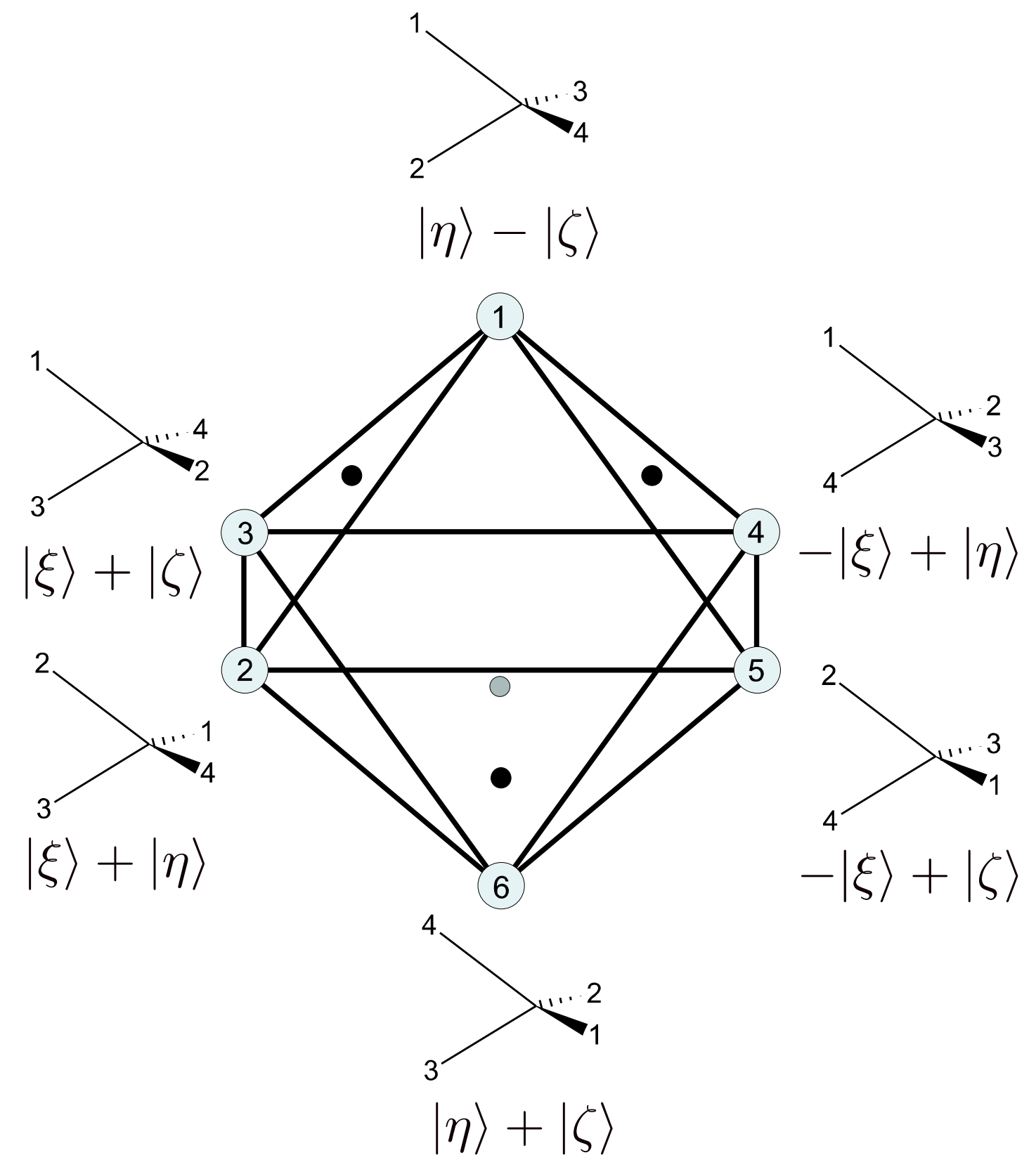

Figure 8: 


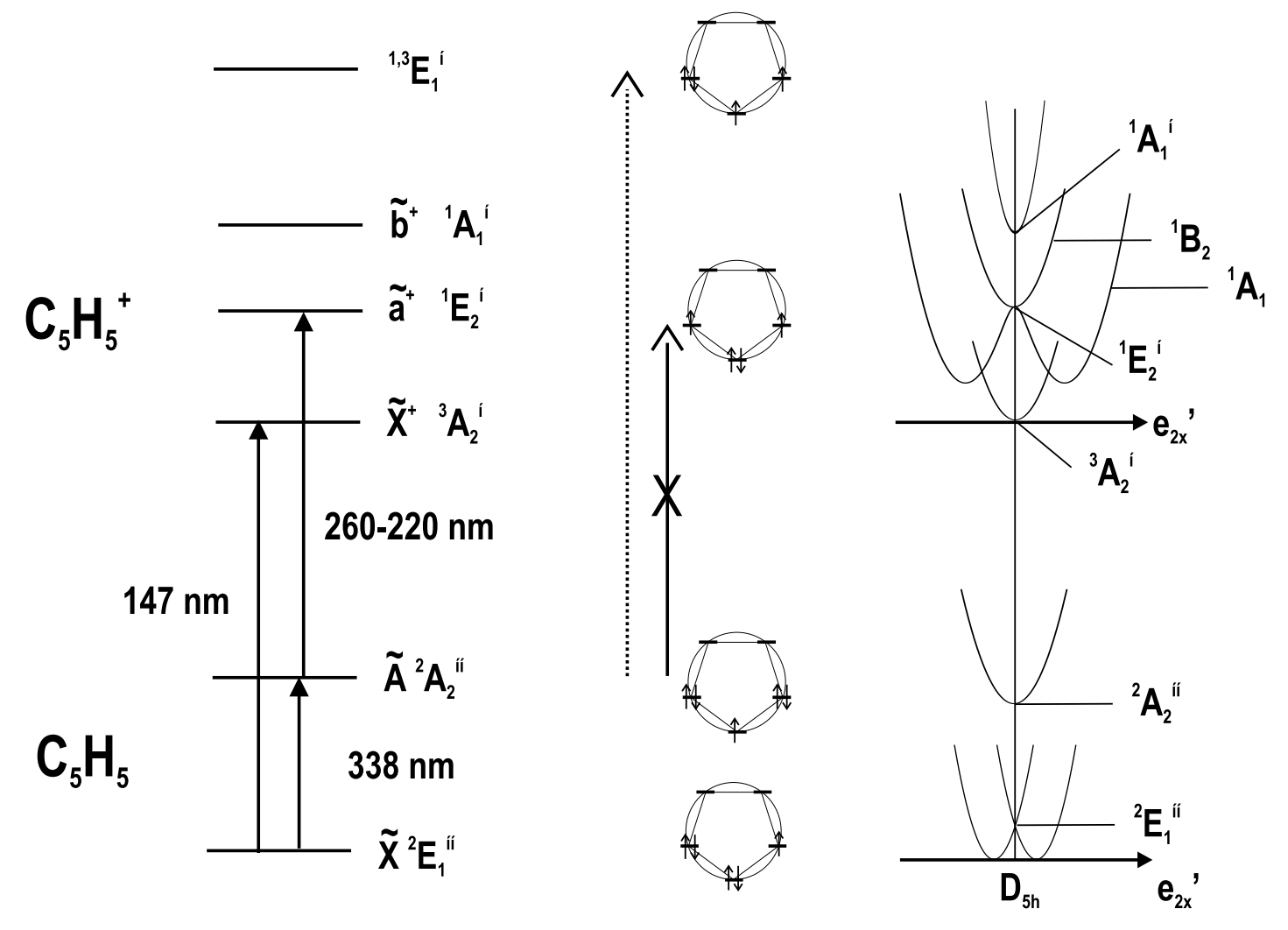

Figure 9: 


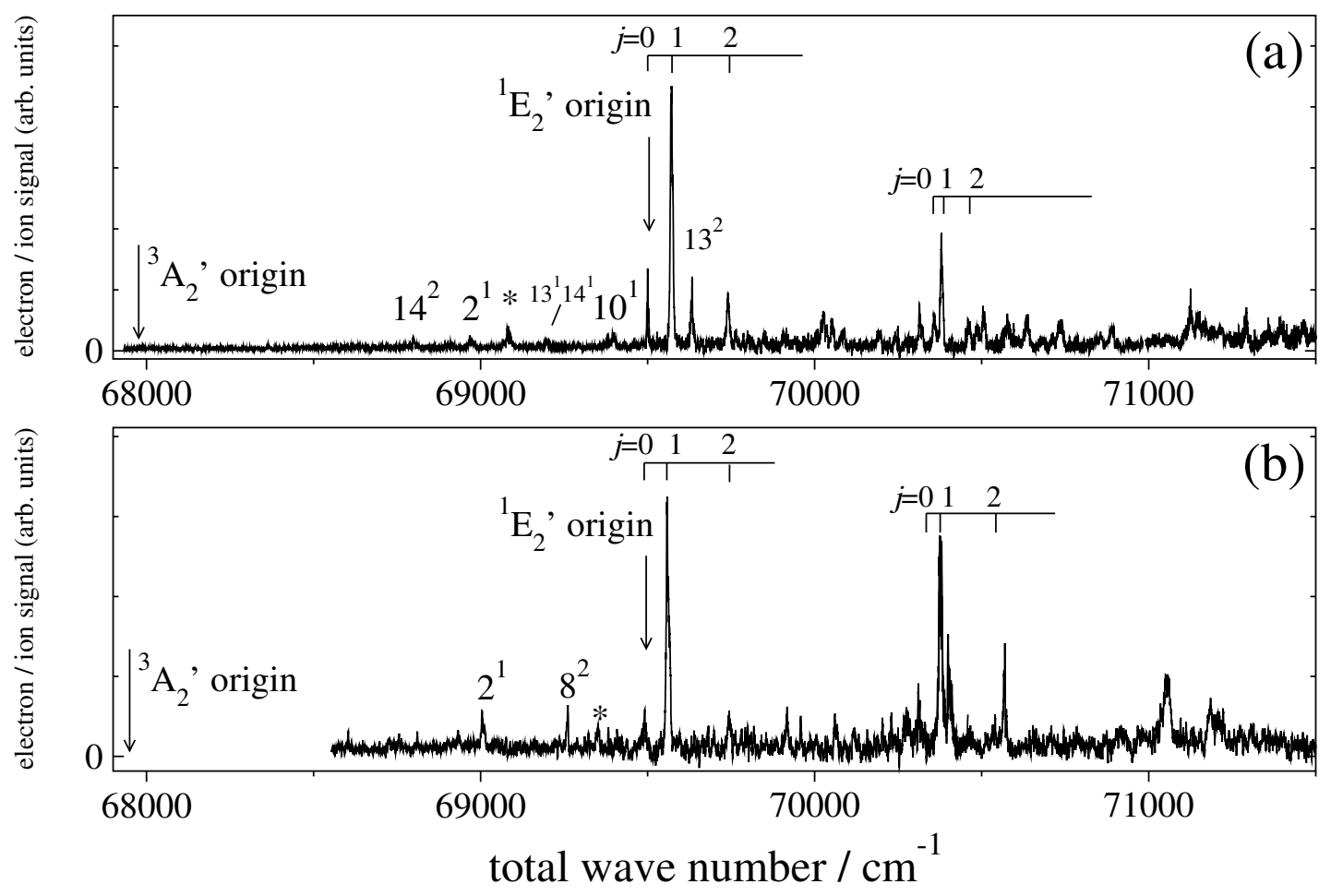

Figure 10: 


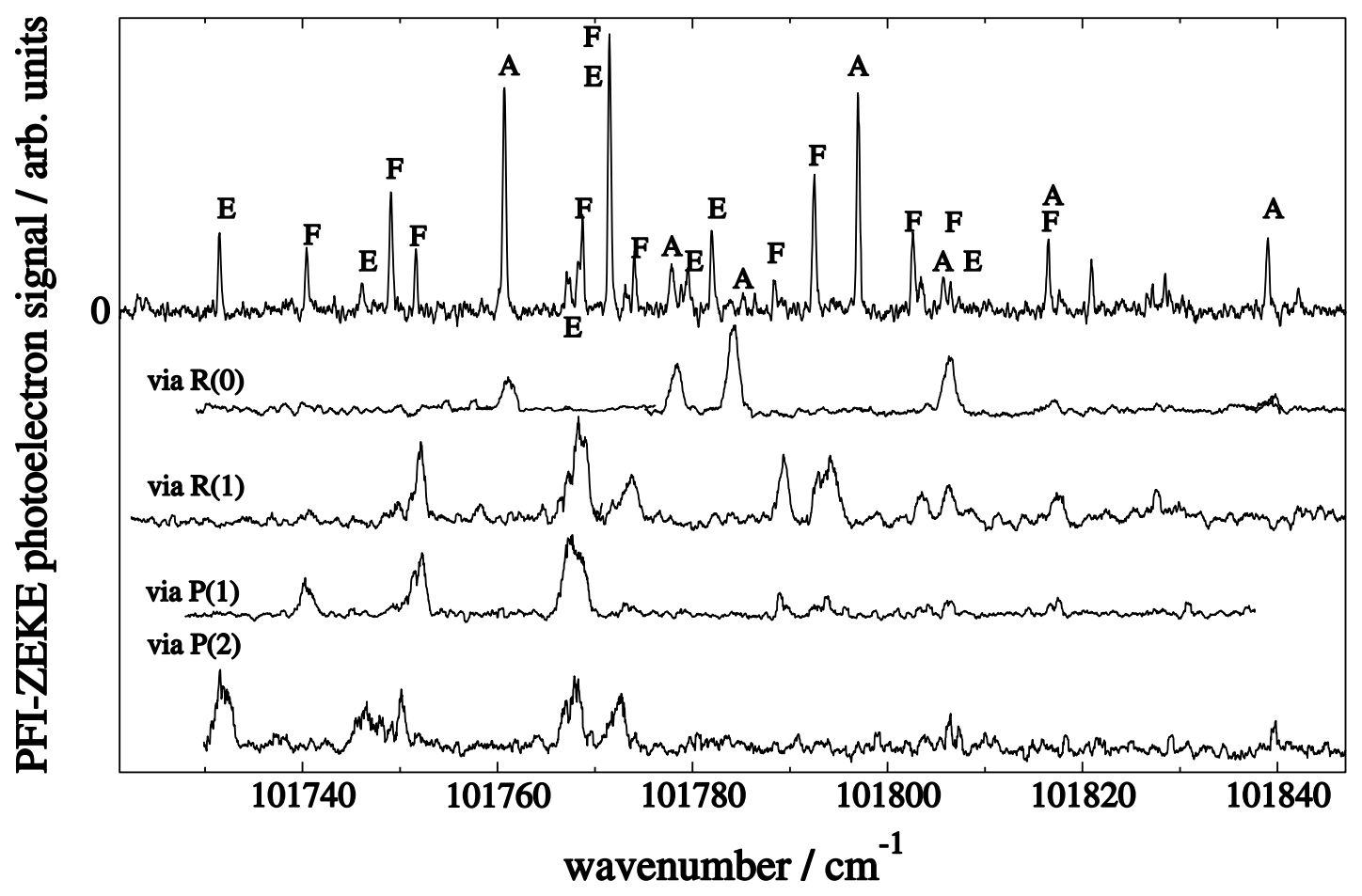

Figure 11: 


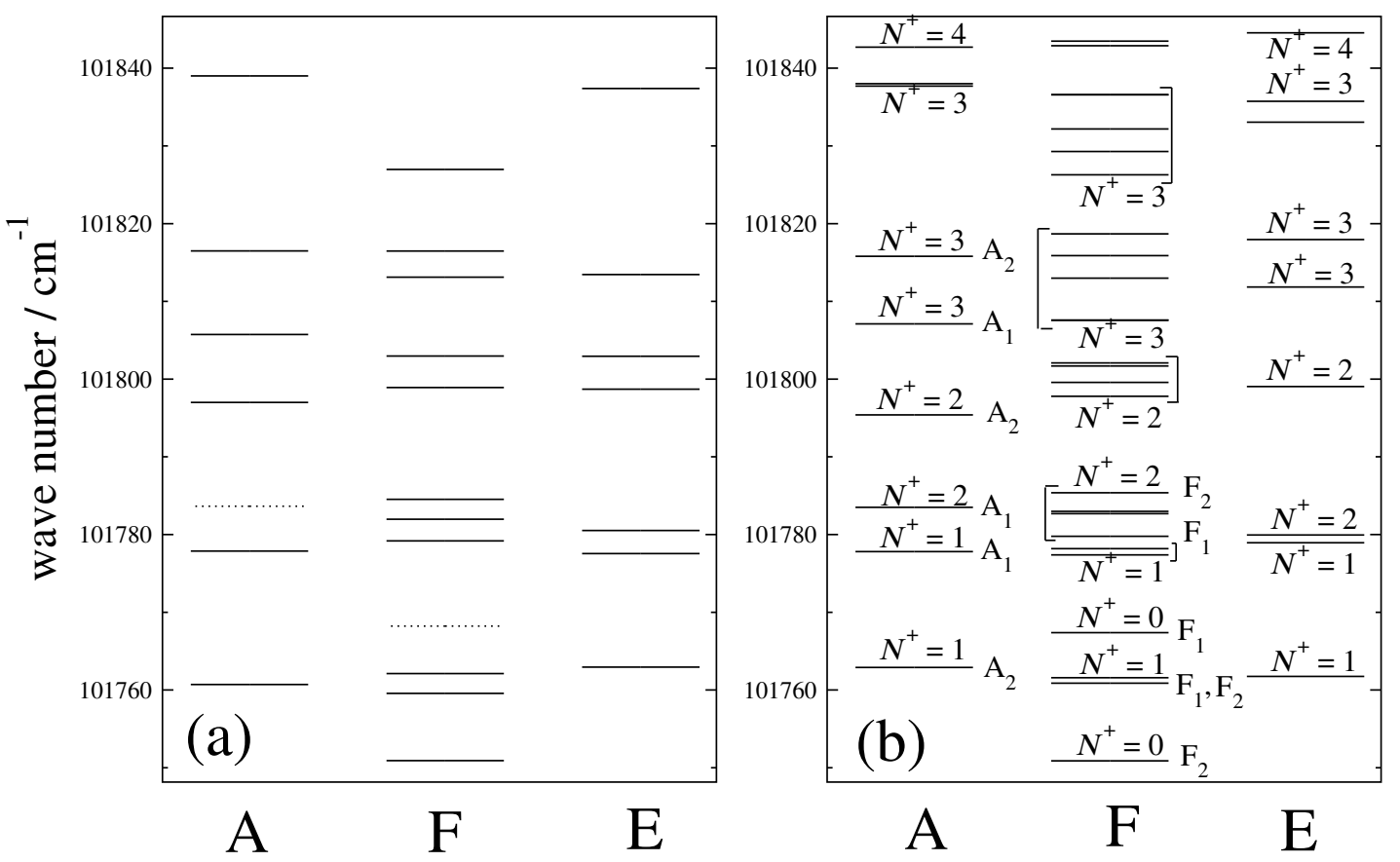

Figure 12: 
$\Gamma_{\mathrm{ev}}$
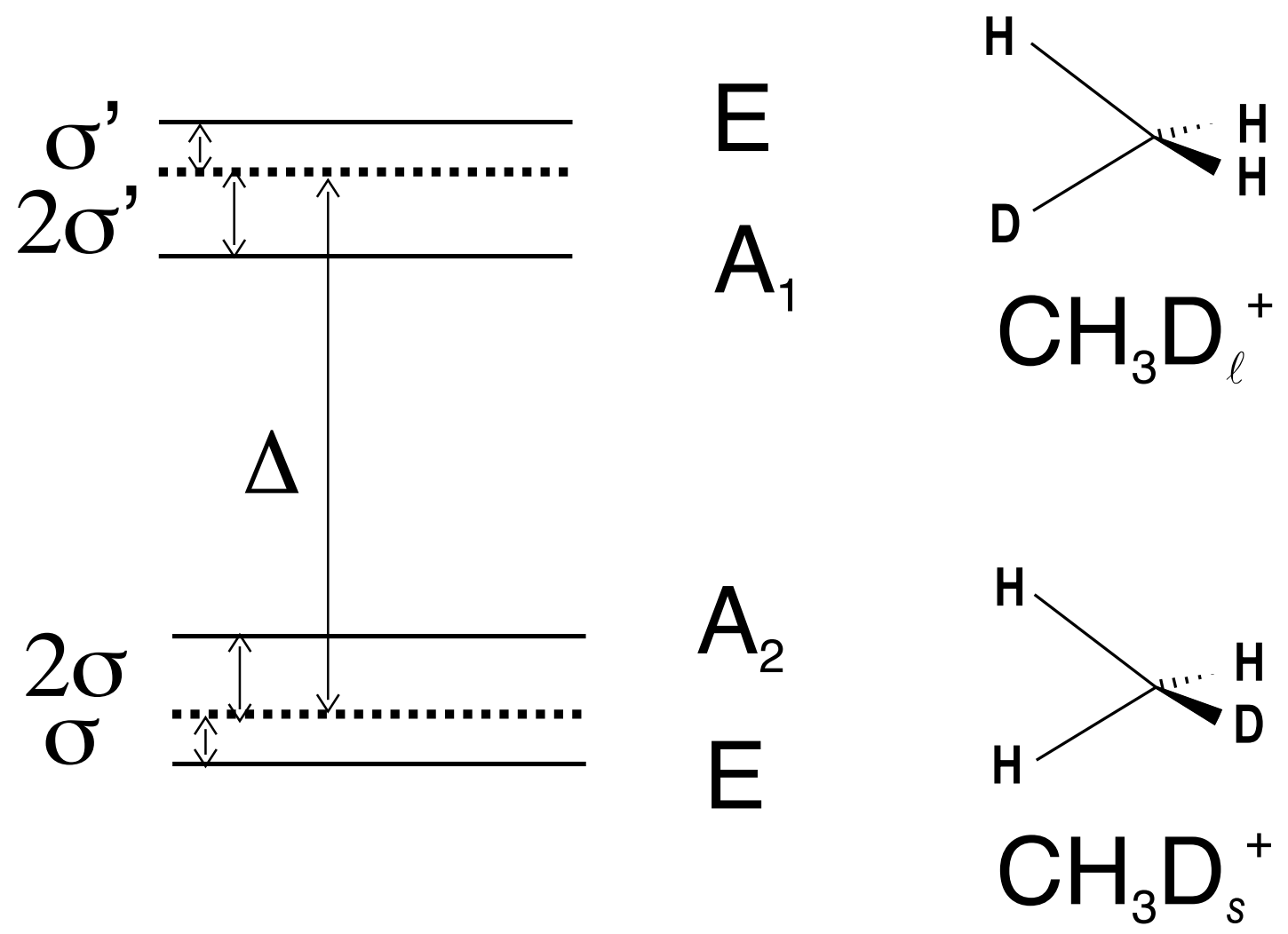

Figure 13: 


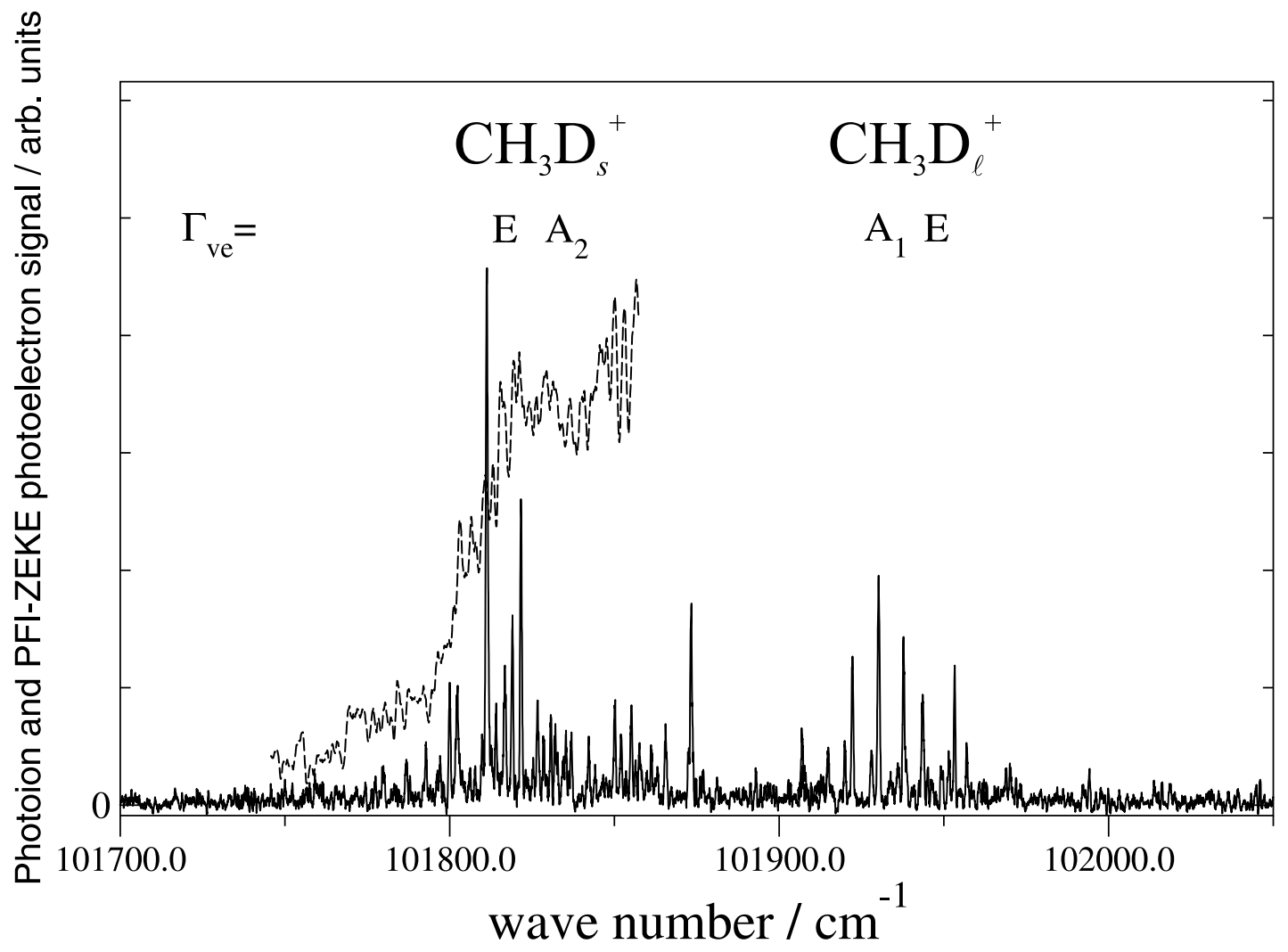

Figure 14: 


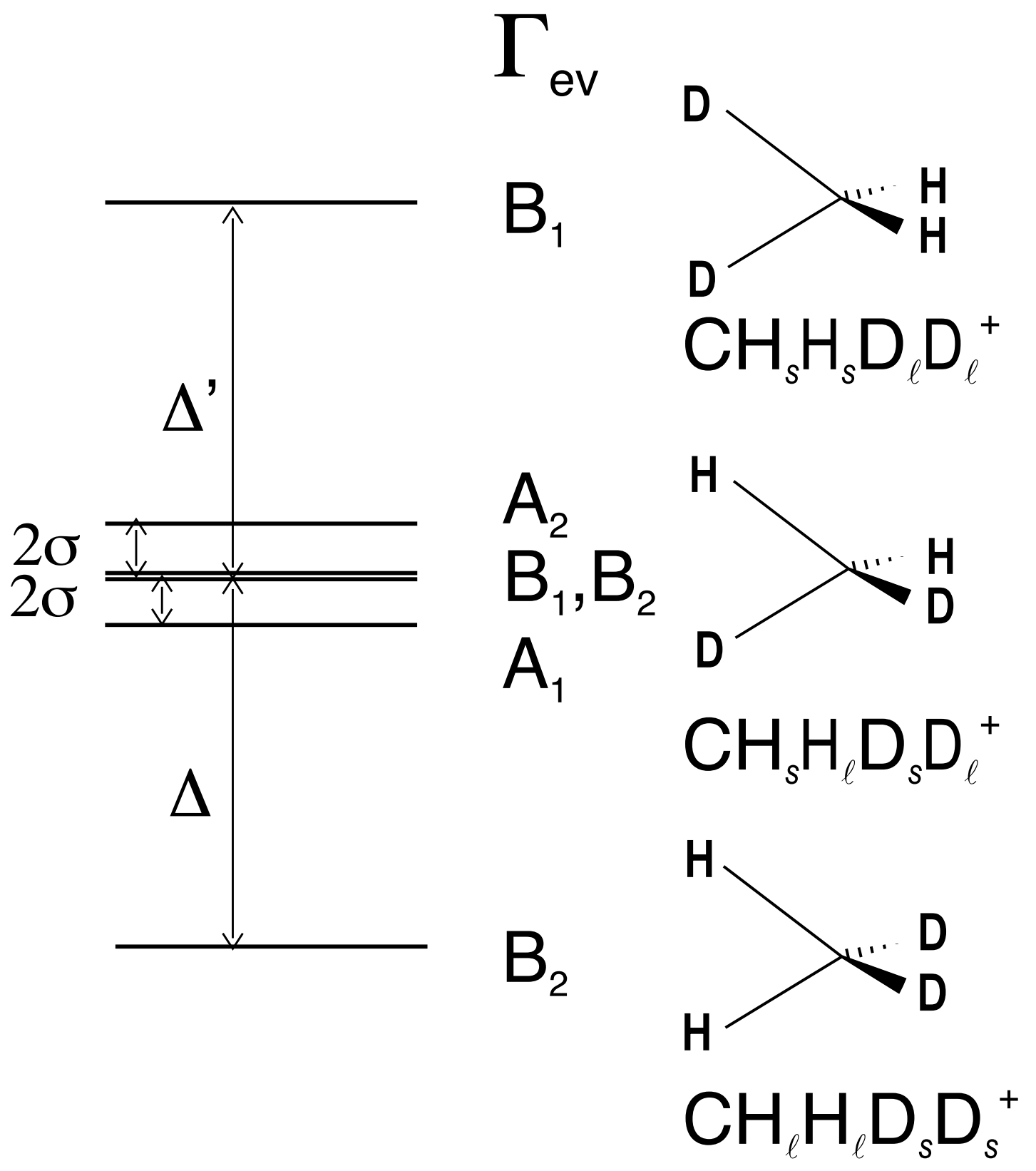

Figure 15: 


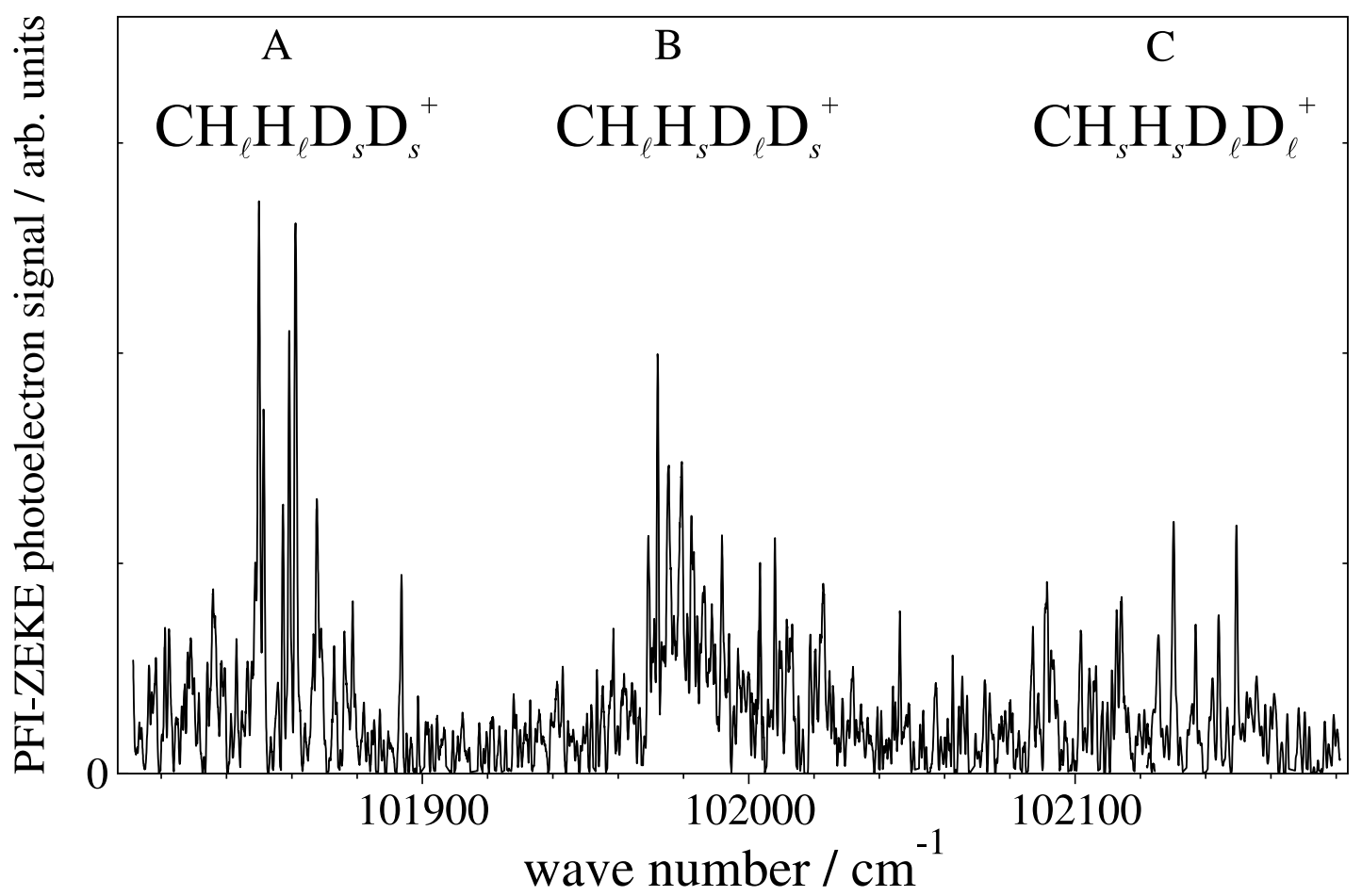

Figure 16: 


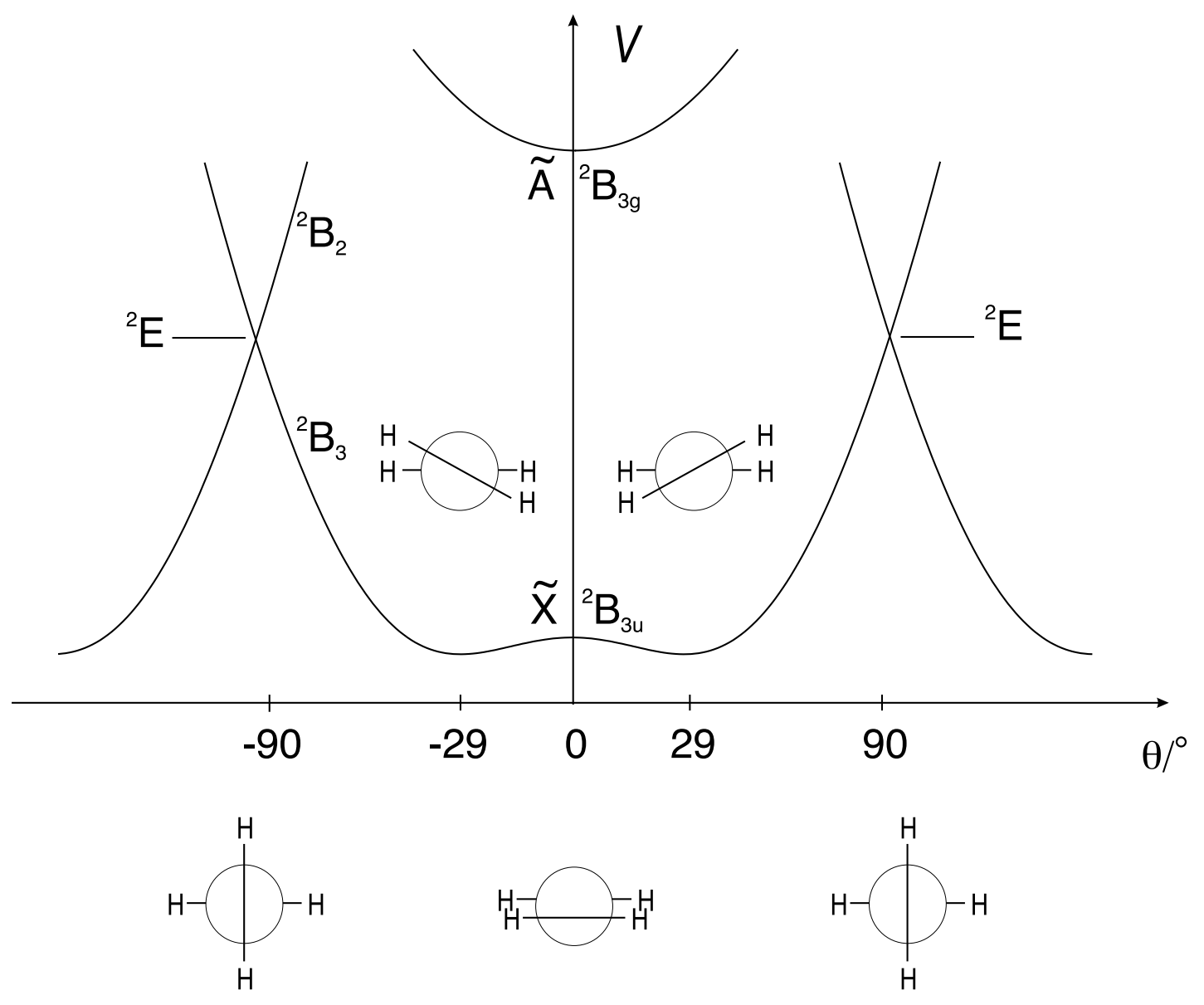

Figure 17: 


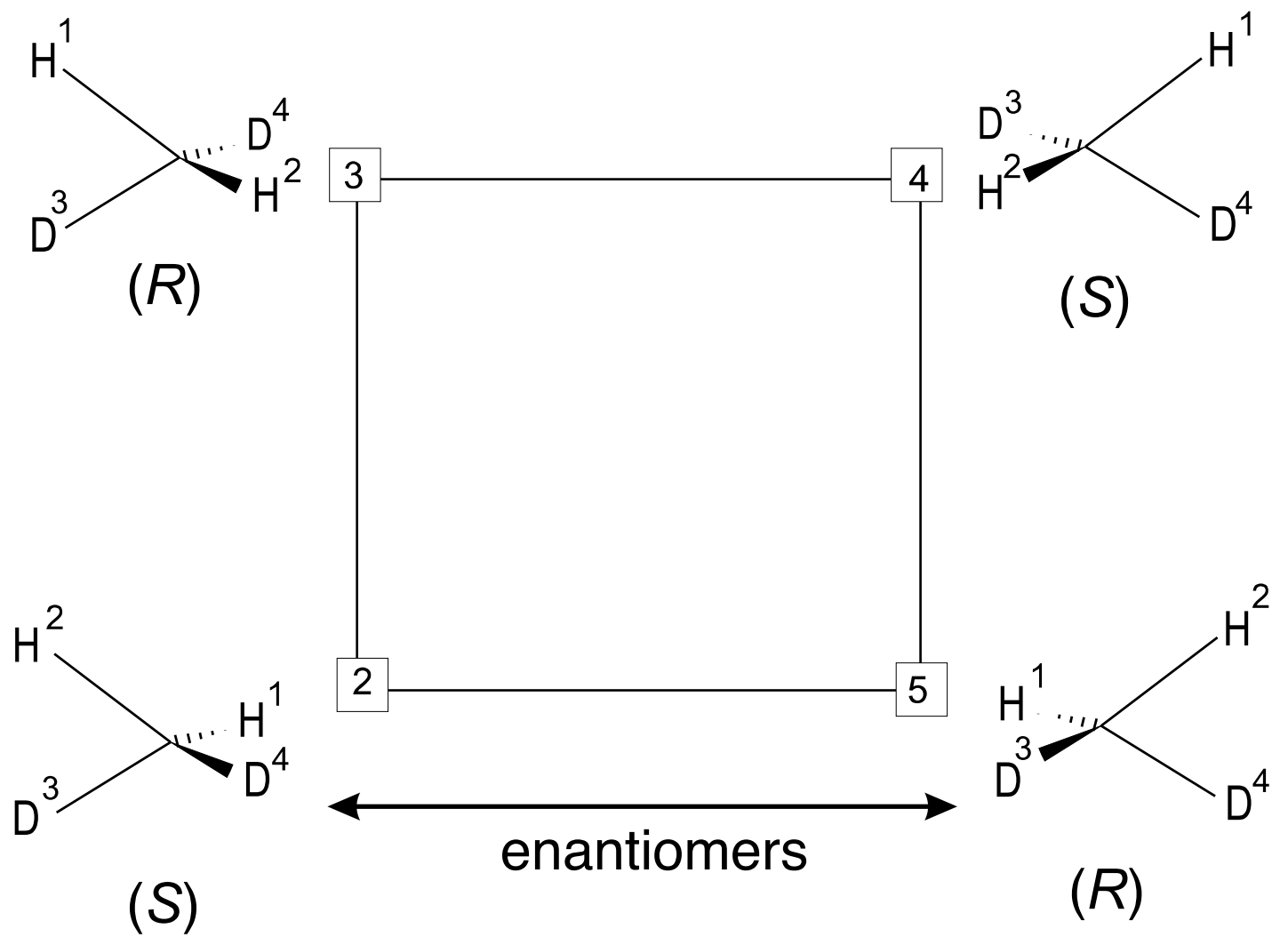

Figure 18: 


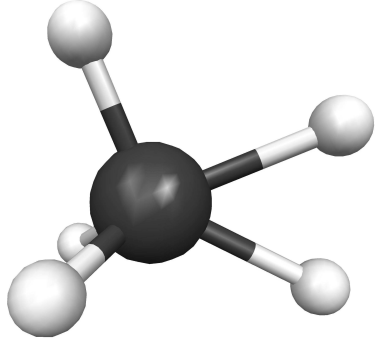

$\mathrm{C}_{\mathrm{s}}(\mathrm{I})$

$\Delta \mathrm{E}_{\mathrm{pot}} \mathrm{l} \quad 0$

$\left(\right.$ hccm $\left.^{-1}\right)$
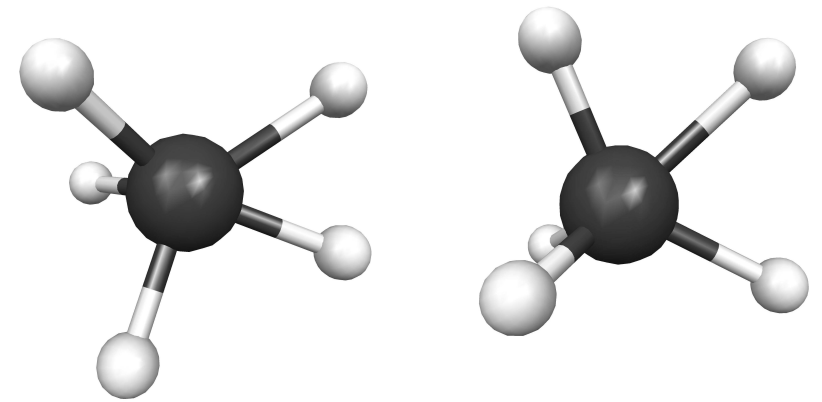

$\mathrm{C}_{\mathrm{s}}(\mathrm{II})$

$\mathrm{C}_{2 \mathrm{v}}$

30

340

Figure 19: 